\title{
Variational quantum simulation of ultrastrong light-matter coupling
}

\author{
Agustin Di Paolo $\odot,{ }^{1, *}$ Panagiotis Kl. Barkoutsos $\odot,{ }^{2}$ Ivano Tavernelli, ${ }^{2}$ and Alexandre Blais ${ }^{1,3}$ \\ ${ }^{1}$ Institut quantique and Département de Physique, Université de Sherbrooke, Sherbrooke, Canada QC J1K 2R1 \\ ${ }^{2}$ IBM Research GmbH, Zurich Research Laboratory, Säumerstrasse 4, 8803 Rüschlikon, Switzerland \\ ${ }^{3}$ Canadian Institute for Advanced Research, Toronto, Ontario, Canada M5G 1M1
}

(Received 27 September 2019; revised 24 June 2020; accepted 14 August 2020; published 3 September 2020)

\begin{abstract}
We propose the simulation of quantum-optical systems in the ultrastrong-coupling regime using a variational quantum algorithm. More precisely, we introduce a short-depth variational form to prepare the ground state of the multimode Dicke model on a quantum processor and present proof-of-principle results obtained using an IBM device. We moreover provide an algorithm for characterizing the ground state by Wigner state tomography. Our work is a first step toward digital quantum simulation of light-matter systems with potential applications to few-impurity spin-boson models.
\end{abstract}

DOI: 10.1103/PhysRevResearch.2.033364

\section{INTRODUCTION}

Quantum simulation is one promising application of quantum processors which aims to circumvent the limitations of classical computers at simulating matter. Recently, a quantumclassical simulation paradigm exploiting variational principles has been introduced [1]. Following this work, many other realizations of what is known as variational quantum algorithm (VQA) have appeared [2-5].

VQAs have some robustness against noise and appear appropriate for noise-intermediate-scale-quantum (NISQ) computation $[4,6,7]$. Although considerable effort has been devoted to solving proof-of-principle problems in chemistry [2-5] and optimization [8], the applicability of VQAs to other domains is a subject of debate and interest $[9,10]$. Here, we use a VQA to simulate interacting light-matter models. More precisely, we study the ground state of a set of two-level atoms coupled to electromagnetic modes, which is of fundamental interest and has important applications in computing and sensing [11-15].

The simplest case corresponds to a two-level atom coupled to a cavity mode, described by the quantum Rabi Hamiltonian

$$
H / \hbar=\frac{\omega_{q}}{2} \sigma^{z}+\omega_{c} a^{\dagger} a+g \sigma^{x}\left(a+a^{\dagger}\right)
$$

Here, $\omega_{q}$ and $\omega_{c}$ are the atomic and the electromagneticmode frequencies, $\sigma^{\mu}(\mu=x, y, z)$ the Pauli matrices. and $a\left(a^{\dagger}\right)$ the annihilation (creation) operator for the oscillator, respectively. If the light-matter coupling constant $g$ is small compared to the systems' frequencies, Eq. (1) reduces to the Jaynes-Cummings Hamiltonian [16]. Then, the terms $\sigma^{+} a$

\footnotetext{
*agustin.di.paolo@usherbrooke.ca

Published by the American Physical Society under the terms of the Creative Commons Attribution 4.0 International license. Further distribution of this work must maintain attribution to the author(s) and the published article's title, journal citation, and DOI.
}

and $\sigma^{-} a^{\dagger}$, where $\sigma^{ \pm}=\left(\sigma^{x} \pm i \sigma^{y}\right) / 2$, lead to an exchange of a single excitation between the atom and the oscillator mode. Provided that $g$ is greater than the decoherence rates of the atom and the cavity, this regime is referred to as strong coupling, and it is widely exploited for quantum-information processing purposes [17].

As $g$ approaches a significant fraction of the atom and cavity frequencies in Eq. (1), or exceeds them, the atom-cavity system enters the ultrastrong-coupling (USC) and deep-strong-coupling (DSC) regimes, respectively $[11,14,15,18,19]$. Now, the counter-rotating terms $\left(\sigma^{+} a^{\dagger}\right.$ and $\sigma^{-} a$ ) in Eq. (1) need to be taken into account. Perturbation theory provides an accurate description for $g$ in the range of $10 \%-30 \%$ of the system's frequencies, but has limited applicability beyond that regime [14]. While an exact analytical solution exists for the energy spectrum of Eq. (1) [20], systems with multiple atoms and/or electromagnetic modes can only be handled numerically.

In the large- $g$ limit, however, the mean cavity-mode photon number and its quantum fluctuations become large enough to make the numerical simulation of many-particle systems difficult or unpractical. This motivates the search for efficient analytical and numerical methods [11-13,21-24] and quantum-simulation algorithms [14,15,19,25-27].

\section{POLARON VARIATIONAL FORM}

We consider the generalization of Eq. (1) to $N$ atoms and $M$ modes, given by [22]

$$
H / \hbar=\sum_{i=1}^{N} \frac{\omega_{q i}}{2} \sigma_{i}^{z}+\sum_{k=1}^{M} \omega_{k} a_{k}^{\dagger} a_{k}+\sum_{i=1}^{N} \sum_{k=1}^{M} g_{i k} \sigma_{i}^{x}\left(a_{k}+a_{k}^{\dagger}\right),
$$

where the constants $\left\{g_{i k}\right\}$ quantify the coupling strength between the $i$ th atom (of frequency $\omega_{q_{i}}$ ) and the $k$ th cavity mode (of frequency $\omega_{k}$ ), referred below to as $k$ mode. For $M=1$, Eq. (2) reduces to the Dicke model, while $N=1$ corresponds to the multimode quantum Rabi model. Digital quantum 
simulation requires the encoding of the bosonic modes into qubit registers. We use a single-excitation-subspace (SES) encoding where the Fock space of a $k$ mode is truncated to a maximum photon number $n_{k}^{\max }$ and represented by a qubit register of size $n_{k}^{\max }+1$ [28-31]. A mapping from the Fock space to the single-excitation subspace is then defined as $\left|n_{k}\right\rangle \rightarrow\left|\tilde{n}_{k}\right\rangle=\left|0_{0} \ldots 0_{n_{k}-1} 1_{n_{k}} 0_{n_{k}+1} \ldots 0_{n_{k}^{\max }}\right\rangle$ for $n_{k} \in$ $\left[0, n_{k}^{\max }\right]$, where the tilde is used hereafter to indicate encoded states and operators. Under SES encoding, quadratic bosonic Hamiltonians lead to next-neighbor interactions at most [28]. Indeed, the $k$-mode annihilation operator maps to $a_{k} \rightarrow \tilde{a}_{k}=\sum_{n_{k}=0}^{n_{k}^{\max }-1} \sqrt{n_{k}+1} \sigma_{n_{k}}^{+} \sigma_{n_{k}+1}^{-}$, where $\sigma_{n_{k}}^{ \pm}$acts on the $n_{k}$ th qubit of the $k$-mode register. The 2-local form of $\tilde{a}_{k}$ relaxes connectivity requirements on the $k$-mode qubit register and leads to a reduced gate count. Other encodings can be found in Refs. [27,32,33].

Finding the ground state $|G\rangle$ of Eq. (2) by means of a VQA requires first to construct a proper variational form $[1,6]$. That is, a unitary $U(\boldsymbol{\theta})$ parametrized by a real-valued vector $\boldsymbol{\theta}$, such that

$$
|\widetilde{G}\rangle \simeq U\left(\theta^{*}\right)|\widetilde{\mathrm{vac}}\rangle,
$$

where $|\widetilde{\mathrm{vac}}\rangle=\left|0_{q}\right\rangle \otimes_{k=1}^{M}\left|\widetilde{0}_{k}\right\rangle$ is the (encoded) noninteracting vacuum state, and $\boldsymbol{\theta}^{*}$ minimizes the energy $E(\boldsymbol{\theta})=$ $\left\langle\widetilde{\mathrm{vac}}\left|U^{\dagger}(\boldsymbol{\theta}) \widetilde{H} U(\boldsymbol{\theta})\right| \widetilde{\mathrm{vac}}\right\rangle$. A convenient choice of $U(\boldsymbol{\theta})$ can be motivated by approximate disentangling transformations for Eq. (2) [22,23], which we refer to as polaron Ansätze. The simplest transformation is obtained for the case of $N=1$, where it is useful to rotate $H \rightarrow H^{\prime}=P^{\dagger} H P$ by means of a qubit-state-dependent displacement of the $k$ modes [23]

$$
P=\prod_{k=1}^{M} \exp \left[g_{k} \sigma^{x}\left(a_{k}+a_{k}^{\dagger}\right) /\left(\omega_{k}+\omega_{q}^{\prime}\right)\right],
$$

where $\omega_{q}^{\prime}$ is a renormalized atom frequency. As illustrated in Appendix A 1, the ground state of $H^{\prime}$ approaches $|\mathrm{vac}\rangle$ in most coupling regimes. Therefore, the state $P|\mathrm{vac}\rangle$ approximates $|G\rangle$ in the laboratory frame.

Exploiting this fact to prepare $|\widetilde{G}\rangle$ on a quantum computer requires compiling $\widetilde{P}$ from single- and two-qubit gates, for instance, using a Trotter decomposition. The need for reducing the Trotter error, however, can lead to quantum circuits of large depth. Moreover, this approach is sensitive to errors arising from imperfect qubit control and noise. As a way around this problem, we propose to leverage the structure of the polaron transformation to obtain a short-depth variational form. We do this by parametrizing the Trotter decomposition of $\widetilde{P}$ and letting the VQA adjust the unitary such that the energy is minimized. The variational form has not only the purpose of discovering short-depth quantum circuits for synthesizing the USC ground state, but also to potentially improving on the disentangling capabilities of Eq. (4).

We construct the variational form by choosing a convenient Trotter decomposition of $\widetilde{P}$, first for the case of $N=1$. We introduce two $k$-mode operators, $\widetilde{X}_{k}^{e}$ and $\widetilde{X}_{k}^{o}$, defined such that $\widetilde{P}=\prod_{k=1}^{M} \exp \left[f_{k} \sigma^{x}\left(\widetilde{X}_{k}^{e}+\widetilde{X}_{k}^{o}\right)\right]$, where $\left\{f_{k}=g_{k} /\left(\omega_{k}+\right.\right.$ $\left.\left.\omega_{q}^{\prime}\right)\right\}$ is a set of constants that will latter play the role of variational parameters. Although $\left[\widetilde{X}_{k}^{e}, \widetilde{X}_{k}^{o}\right] \neq 0, \widetilde{X}_{k}^{e}$ and $\widetilde{X}_{k}^{o}$ are, respectively composed of commuting terms that act on even and odd sites of the $k$-mode qubit register, the 2-local form of the encoded bosonic operators leads to an efficient implementation of the Trotter-expanded unitary

$$
\widetilde{P}_{\boldsymbol{d}} \simeq \prod_{k=1}^{M} \prod_{s=1}^{d_{k}} \exp \left(\frac{f_{k}}{d_{k}} \sigma^{x} \widetilde{X}_{k}^{e}\right) \exp \left(\frac{f_{k}}{d_{k}} \sigma^{x} \widetilde{X}_{k}^{o}\right),
$$

where $d_{k}$ is the number of Trotter steps that may vary with the $k$-mode index. As shown in $\mathrm{A}$, the exponentials in this equation exactly factorize into a product of $n_{k}^{\max }$ controlledexchange gates acting on next-neighbor qubits of the $k$-mode register, with the atom register being the control. The implementation of Eq. (5) requires $n_{k}^{\max } \times d_{k}$ such gates per $k$ mode, adding to a gate count of $\sum_{k=1}^{M} n_{k}^{\max } d_{k}$. This number grows linearly with the number of $k$ modes, their Fock-space dimension, and the order of the Trotter expansion (Trotter depth). Since Eq. (5) parallelizes over the $k$ modes, its depth does not scale with $M$.

For $N>1$, our variational form incorporates blocks of the form of Eq. (5) where the operator $\sigma^{x} \rightarrow \sigma_{i}^{x}$ is now indexed by $i \in[1, N]$ (see Appendix A 3), arriving at

$$
\text { Varform }=\prod_{i=1}^{N} \prod_{k=1}^{M} \prod_{s=1}^{d_{i k}} \exp \left(\frac{f_{i k}^{s}}{d_{i k}} \sigma_{i}^{x} \widetilde{X}_{k}^{e}\right) \exp \left(\frac{f_{i k}^{s}}{d_{i k}} \sigma_{i}^{x} \widetilde{X}_{i k}^{o}\right),
$$

where the coefficients $f_{k} \rightarrow f_{i k}^{s}$ are variational parameters that depend on the Trotter step $s \in\left[1, \ldots, d_{i k}\right]$. Additionally, $f_{i k}^{s}$ can also be made a function of the $k$-mode photon number, i.e., $f_{i k}^{s} \rightarrow f_{i k}^{s}\left(n_{k}\right)$. As argued below, this trades shorter circuits for longer optimization runtime.

Additional details apply, however, between the cases $N=$ 1 and $N>1$. Indeed, $N>1$ requires Eq. (6) to be complemented by a short-depth variational form on the atoms' registers. This extra step initializes the polaron variational circuit to the state $\left|\widetilde{\operatorname{vac}}^{\prime}\right\rangle=\prod_{k=1}^{M}\left|\psi_{a}\right\rangle\left|\tilde{0}_{k}\right\rangle$, where $\left|\psi_{a}\right\rangle$ is an entangled state of the atoms, determined by an auxiliary optimization loop.

\section{VARIATIONAL QUANTUM ALGORITHM}

Figure 1 shows the results for the (a) single- and (b) twomode Rabi Hamiltonian, and (c) single- and (d) two-mode Dicke model for $N=2$. The atoms and $k$-modes frequencies are set to $\omega_{q_{i}}=\omega_{k} \equiv \omega$ and $g_{i k} \equiv g$ is swept in $[0, \omega]$. The resonance condition favors entanglement between the atoms and cavity modes due to the energetically allowed exchange of excitations. To quantify the VQA performance, we define the error metric $\Delta_{\text {en }}=\left|\left(E_{\mathrm{vqe}}-E_{\text {en }}\right) / E_{\text {en }}\right|$ accounting for the relative difference between the ground-state energy found by the VQA, $E_{\mathrm{vqe}}$, and the energy of the encoded ground state $E_{\text {en }}$. Additionally, $\Delta_{\text {ex }}=\left|\left(E_{\text {en }}-E_{\text {ex }}\right) / E_{\text {ex }}\right|$ quantifies the difference between $E_{\mathrm{en}}$ and the numerically exact groundstate energy. We evaluate $\Delta_{\mathrm{en}}$ and $\Delta_{\mathrm{ex}}$ as a function of $g / \omega$ for circuits with $d_{i k}=d$. The chosen Fock space truncation (see figure caption) leads to a small number of qubits while ensuring a relatively small $\Delta_{\mathrm{ex}}$. This choice seeks to reduce the quantum-hardware resources needed for simulation.

Results in Fig. 1(a) show that errors $\Delta_{\text {en }}$ below $1 \%$ are achieved by circuits containing three variational parameters $(d=3)$. Same accuracy is obtained for circuits with $d=2$ 

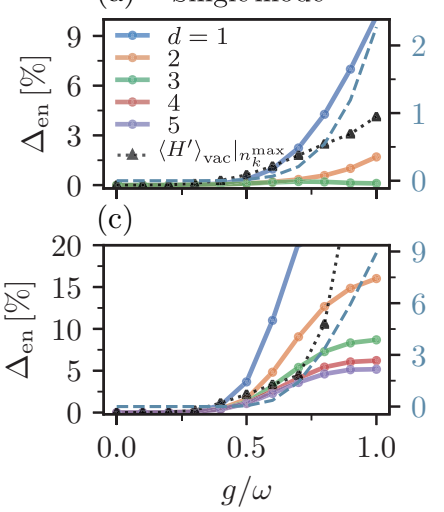

(a) Single mode

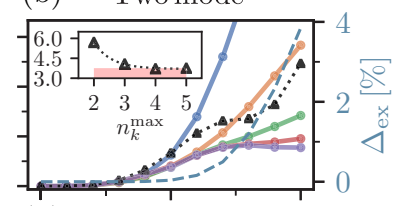

(d)

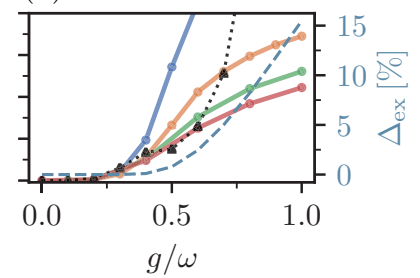

(b) Two mode

FIG. 1. Ground-state-energy estimation for the single- and twomode Rabi and Dicke models in resonance. The Fock-space truncation in (a)-(d) is set to $n_{k}^{\max }=3,3,5$, and 4 corresponding to $5,9,8$, and 12 qubits, respectively. The legend is shared between all panels, although (a) and (d) display results only for $d_{i}<4$ and $d_{i k}<5$, respectively. We show the error metrics $\Delta_{\text {en }}$ (left scale) and $\Delta_{\text {ex }}$ (light blue dashed line and right scale), along with $\left.\left\langle H^{\prime}\right\rangle_{\text {vac }}\right|_{n_{\mathrm{k}}^{\max }}$ (triangular markers). The inset in (b) shows $\left.\left\langle H^{\prime}\right\rangle_{\text {vac }}\right|_{n_{\mathrm{b}}^{\max }}$ converging to a $4 \%$ in the limit of large $n_{k}^{\max }$ (pink baseline) for $g / \omega=0.8$. Simulations do not include noise and are done using QISKIT [34].

if additional parameters, dependent on the $k$-mode photon number, are incorporated (not shown). For $d>1$, the energy of the variational Ansatz is significantly lower than the expectation value $\left.\left\langle H^{\prime}\right\rangle_{\text {vac }}\right|_{n_{k}^{\max }}$ of Eq. (2) on the state $\left.P|\mathrm{vac}\rangle\right|_{n_{k}^{\max }}$ within a truncated Fock space. This indicates that the VQA leverages the Trotter error to outperform the full polaron Ansatz under the same Fock-space restrictions and with very low circuit depth. The energy of the variational state also falls below $\left.\left\langle H^{\prime}\right\rangle_{\mathrm{vac}}\right|_{n_{k}^{\max } \rightarrow \infty}$ in the full range of $g / \omega \in[0,1]$ (not shown). This suggests that refining Eq. (4) to allow for a more extensive parametrization can lead to a better approximation for the ground state of Eq. (1). However, while the optimal parametrization of Eq. (4) is found efficiently by semianalytical methods, the parameters of our variational form are determined by a quantum algorithm. Finally, we note that the error metric $\Delta_{\text {ex }}$ remains below $\sim 2 \%$ in all cases.

We observe a similar behavior for the two-mode simulations in Fig. 1(b), although $\Delta_{\text {en }}$ increases to $\sim 2.5 \%$ for $d=4$. Same accuracy is reached for circuits with $d=2$ when more variational parameters are introduced (not shown). We find that the accuracy limit is due to truncation errors and the disentangling capabilities of the polaron Ansatz. Increasing the number of two-level atoms in the model, while keeping the number of qubits of the order of 10 , leads to the results in Figs. 1(c) and 1(d) for which we find a maximum error of $\Delta_{\text {en }} \simeq 5 \%$ for $d=5$ in the first case, and of $\Delta_{\text {en }} \simeq 8 \%$ for $d=4$ in the second case. These results, however, are limited by Fock-space truncation errors and can be improved by increasing the number of qubits in the simulations. It is worth noticing that, similarly to the case of $N=1$, these variational circuits outperform the polaron Ansatz significantly for the same conditions.

The performance of the variational form may be improved further by means of simple modifications. For instance, a layer

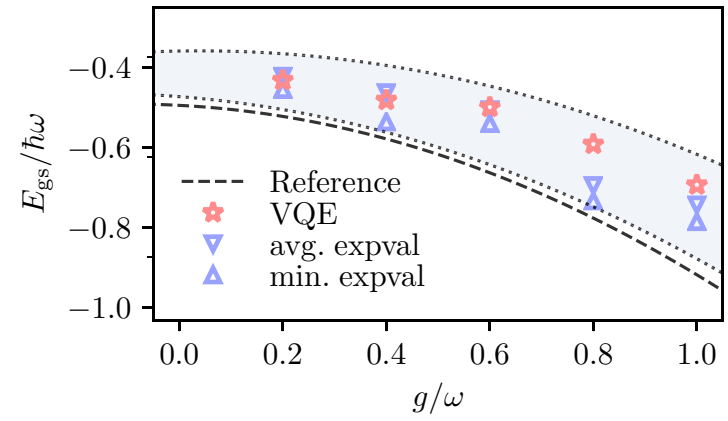

FIG. 2. Variational simulation of the Rabi model on a quantum processor. Shown is the ground-state energy as a function of $g$ in units of $\omega$. The light blue bands enclose the range of results that are expected for 150 SPSA trials with levels of noise in the order of 0.1 and 1.0 relative to calibrated values (dotted lines). The black dashed line corresponds to the encoded-ground-state energy obtained by exact diagonalization. The star-shaped markers are the result of VQA runs for up to 150 SPSA trials on the quantum device. The pointingup (pointing-down) triangular markers are the minimum (average) expectation value on quantum hardware of states that have been previously optimized in the classical processor. The dispersion of such values is due to fluctuations in the level of noise of the quantum device between runs. Further details are provided in Appendix B.

of a hardware-efficient (HE) gates [3] could be appended after a Trotter step. Ideally, gates on such HE layers should conserve the number of excitations in the $k$-mode registers [30]. Generalizations of Eq. (4) are also a possibility [35]. Circuits of larger depth and number of qubits could also benefit from quantum devices tailored to compile the polaron Ansatz using fewer gates, as illustrated in Appendix E.

The results of Fig. 1 suggest that the polaron variational form is a promising tool for investigating the USC ground state in near-term quantum devices. We now implement the aforementioned strategy in currently available quantum hardware. We use the IBM Q Poughkeepsie chip via the open-source framework QISKIT, ${ }^{1}$ taking advantage of the builtin SPSA optimizer [3,36] and readout-error-mitigation techniques [34]. We use three qubits for the quantum simulation, two of them encoding the bosonic mode. The ground-state energies found this way, shown in Fig. 2 (star-shaped data points), are in good qualitative agreement with the theoretical estimations.

We find that the main limitations on the accuracy of the VQA are due to noise in the quantum processor and the performance of simultaneous-perturbation-stochasticapproximation (SPSA). To investigate the effect of the latter against the former, we perform the VQA on a desktop computer, assuming a larger number of optimization steps and the calibrated noise model of the device. This produces a set of variational states with optimal parametrization. We then evaluate the energy expectation value of such states on the

${ }^{1}$ IBM Q, Qiskit are trademarks of International Business Machines Corporation, registered in many jurisdictions worldwide. Other product or service names may be trademarks or service marks of IBM or other companies. 
quantum processor. The result of this experiment (triangularshaped data points) reach better accuracies than those obtained by means of the quantum-classical VQA, indicating that noise in the quantum processor prevents SPSA from achieving high-accuracy solutions in a reasonable number of steps (150 trials). By controlling the level of noise in classical simulation, we find that quantum-classical VQA solutions with $\Delta_{\text {en }} \sim 1 \%-2 \%$ and 150 SPSA trials could be achieved with noise levels one order of magnitude smaller than the present value. Note that in absence of noise, the number of optimizer steps required to reach numerical accuracy with respect to the reference value is very small in comparison, below 30 in the entire $g / \omega \in[0,1]$ range.

\section{STATE TOMOGRAPHY AND THE EFFECT OF NOISE}

Following this proof-of-principle demonstration, we present an alternative method for characterizing the prepared ground state. This technique could be useful to probe entanglement metrics and to distinguish between nearly degenerate states. The latter situation appears, for instance, approaching the DSC regime [20,37]. We thus introduce the joint Wigner function for a set of $N$ qubits and $M$ modes as

$$
W_{l}(\boldsymbol{\alpha})=\operatorname{Tr}\left[\rho \sigma_{1}^{l_{1}} \ldots \sigma_{N}^{l_{N}} 2^{M} \Pi(\boldsymbol{\alpha}) / \pi^{M}\right],
$$

generalizing the definition given in Ref. [38] for $N=$ $M=1$. Here, $\left\{\sigma_{i}^{l_{i}}, l_{i} \in[0, x, y, z]\right\}$ are the Pauli matrices for the $i$ th atom with $\sigma_{i}^{0}=\mathbb{1} . \Pi(\boldsymbol{\alpha})=D(\boldsymbol{\alpha}) \Pi D^{\dagger}(\boldsymbol{\alpha})$, where $\boldsymbol{\alpha}=\left(\alpha_{1}, \ldots, \alpha_{M}\right)$ is a joint-parity operator with $\Pi=$ $\prod_{k=1}^{M} \exp \left(i \pi a_{k}^{\dagger} a_{k}\right)$ and $D(\boldsymbol{\alpha})=\prod_{k=1}^{M} \exp \left(\alpha_{k} a_{k}^{\dagger}-\alpha_{k}^{*} a_{k}\right)$ for $\alpha_{k} \in \mathbb{C}$. The inversion of Eq. (7) allows for the reconstruction of $\rho$ [38].

Expanding Eq. (7) using the SES encoding, we arrive at

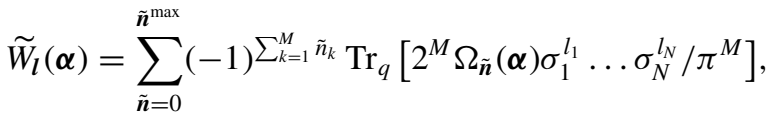

where $\operatorname{Tr}_{q}$ is the trace operator over the atoms and $\Omega_{\tilde{n}}=$ $\left\langle\tilde{n}_{1} \ldots \tilde{n}_{M}\left|\widetilde{D}^{\dagger}(\boldsymbol{\alpha}) \tilde{\rho} \widetilde{D}(\boldsymbol{\alpha})\right| \tilde{n}_{1} \ldots \tilde{n}_{M}\right\rangle . \widetilde{W}_{l}(\boldsymbol{\alpha})$ can be sampled by executing a relatively simple quantum circuit. While tomography gates correspond to single-qubit rotations applied to the atoms' registers, tomography requires the implementation of $\widetilde{D}(\boldsymbol{\alpha})$ on the $k$-modes' registers. Fortunately, the displacement operators can be easily implemented by a sequence of oneand two-qubit gates derived from a Trotter decomposition similar to that of the polaron transformation. Appendix $\mathrm{C}$ includes further details.

We numerically demonstrate this approach for $N=M=1$ and $g / \omega=1$ in resonance. Figure 3 shows $\widetilde{W}_{\sigma^{z}}(\alpha)$ for an eight-qubit $k$-mode register in absence of noise. The result is compared to $W_{\sigma^{z}}$, which is not affected by Trotter and Fock-space truncation errors. We observe a good qualitative agreement between the two distributions, even for a few as two Trotter steps. While large Trotter depths alleviate discrepancies, the implementation accuracy of $\widetilde{W}_{\sigma^{z}}(\alpha)$ remains bounded due to Fock-space truncation errors. In a quantum device, noise can lead to nonunitary errors for circuits based on Trotter decompositions, possibly having an impact on $\widetilde{W}_{\sigma^{z}}(\alpha)$. Performance metrics such as those introduced in Ref. [39]
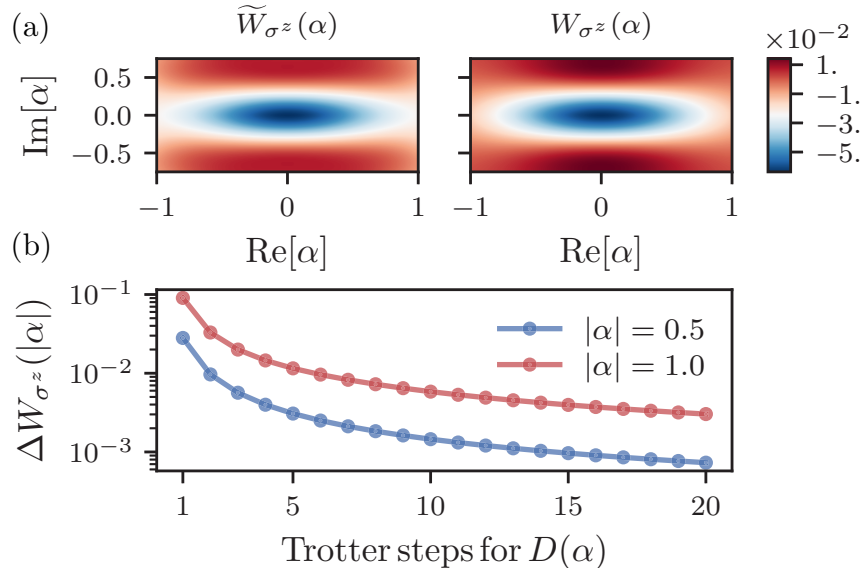

FIG. 3. Reconstruction of the joint Wigner function for the resonant Rabi model for $g / \omega=1$. (a) (Left) Sampled distribution $\widetilde{W}_{\sigma^{z}}(\alpha)$ for an eight-qubit $k$-mode register and two Trotter steps per imaginary and real components of $\widetilde{D}(\boldsymbol{\alpha})$ in absence of noise. (Right) Numerically exact result $W_{\sigma^{z}}(\alpha)$. (b) Effect of the Trotter order of $D(\boldsymbol{\alpha})$ on the reconstructed distribution. The error metric is defined as $\Delta W_{\sigma^{z}}(|\alpha|)=\sqrt{\int_{\left|\alpha^{\prime}\right|=0}^{\left|\alpha^{\prime}\right|=|\alpha|}\left[\widetilde{W}_{\sigma^{z}}\left(\alpha^{\prime}\right)-W_{\sigma^{z}}\left(\alpha^{\prime}\right)\right]^{2} d^{2} \alpha^{\prime}} / \mathcal{N}_{|\alpha|}$, where $1 / \mathcal{N}_{|\alpha|}$ is a normalization factor. This metric scales with the Trotter error as $|\alpha|^{2} / d$, where $d$ being the Trotter depth.

could be used to quantify and improve the fidelity of the state-characterization circuits.

Finally, we discuss the effect of noise channels on the performance of the VQA. It is worth highlighting that SES encodings allow to detect damping errors of the form $\left|\tilde{n}_{k}\right\rangle \rightarrow$ $\left|\widetilde{0}_{k}\right\rangle$ by joint-parity measurements of the $k$-mode register. Postselection can then significantly reduce the impact of noise on the VQA [40]. The downsides of using SES encodings reside in two main points. First, it trades shorter quantumcircuit depths for a relatively large qubit overhead compared to other encodings [32,33]. Second, noise channels that do not conserve the number of excitations can become dominant for large qubit arrays (see Appendix D), as the size of the complement of the SES grows exponentially with the number of qubits. These observations are, however, applicable to other SES proposals [28-31].

\section{CONCLUSION AND OUTLOOK}

In conclusion, we introduced a short-depth and fewparameter variational form to study the ground state of $N$ atoms and $M$ electromagnetic modes. We found that such a variational circuit can approximate the ultrastrong-coupling ground state with very good accuracy. We implemented a proof-of-principle demonstration on an IBM quantum processor. Finally, we used Wigner state tomography to characterize the ground state and discussed the impact of noise on the VQA. As the light-matter Hamiltonian is formally identical to the few-impurity spin-boson model, we envision applications ranging from quantum optics to problems in condensedmatter physics. We anticipate that reaching quantum advantage using our approach would require quantum-hardware performance significantly beyond what is currently available. 
Our work is, however, a first step toward fully digital quantum simulation of interacting light-matter models.

\section{ACKNOWLEDGMENTS}

We thank C. Leroux, A. C. Y. Li, and D. Poulin for insightful discussions, and R. Chen and M. Pistoia for support. P.K.B. and I.T. acknowledge financial support from the Swiss National Science Foundation (SNF) through the Grant No. 200021-179312. This work was undertaken thanks in part to funding from NSERC and the Canada First Research Excellence Fund.

\section{APPENDIX A: POLARON TRANSFORMATION AND QUANTUM-CIRCUIT COMPILATION OF THE POLARON VARIATIONAL FORM}

\section{Polaron transformation for the multimode quantum Rabi Hamiltonian}

In this section, we study the effect of the polaron transformation [Eq. (4)] on the multimode Rabi Hamiltonian $[N=1$ in Eq. (2)]. We first consider a slightly more general unitary of the form

$$
P_{f}=\exp \left[\sigma^{x} \sum_{k=1}^{M} f_{k}\left(a_{k}-a_{k}^{\dagger}\right)\right],
$$

where $\left\{f_{k}\right\}$ are real parameters to be determined below. By transforming $H \rightarrow H_{f}^{\prime}=P_{f}^{\dagger} H P_{f}$, where $\boldsymbol{f}=\left(f_{1}, \ldots, f_{M}\right)$, we arrive at

$$
\begin{aligned}
H_{f}^{\prime} / \hbar= & \frac{\omega_{q}^{\prime}}{2} \sigma^{z} q_{-f}^{\dagger} q_{f}+\sum_{k=1}^{M} \omega_{k} a_{k}^{\dagger} a_{k}+g_{k}^{\prime} \sigma^{x}\left(a_{k}+a_{k}^{\dagger}\right) \\
& +E_{f} .
\end{aligned}
$$

Here, $\omega_{q}^{\prime}=\omega_{q} e^{-2 f \cdot f}$ is a renormalized frequency for the two-level atom, $g_{k}^{\prime}=g_{k}-\omega_{k} f_{k}$ are new light-matter coupling parameters and $E_{f}=\sum_{k=1}^{M} \omega_{k} f_{k}^{2}-2 g_{k} f_{k}$ is an energy constant. We have moreover defined the operator $q_{f}=$ $\exp \left(2 \sigma^{x} \sum_{k=1}^{M} f_{k} a_{k}\right)$.

The parameters $f_{k}$ in Eq. (A2) can reduce the effective light-matter coupling strength $g_{k}^{\prime}$ and thus make the ground state of such a Hamiltonian closer to that of the noninteracting case. This, however, holds if the higher-order corrections to $\sigma^{z}$ from the operator $q_{-f}^{\dagger} q_{f}$, which mixes the $k$ modes, can be made small at the same time. This leads to a compromise for the best value of $\boldsymbol{f}$ that can be resolved by optimization of such a parameter [23,41]. More precisely, assuming that the ground state of Eq. (A2) results close to vacuum, minimizing the ground-state energy $E_{f}-\omega_{q}^{\prime} / 2$ leads to the optimal condition $f_{k}=g_{k} /\left(\omega_{k}+\omega_{q}^{\prime}\right)$ and to the implicit equation

$$
\omega_{q}^{\prime}=\omega_{q} e^{-2 \sum_{k=1}^{M}\left[g_{k} /\left(\omega_{k}+\omega_{q}^{\prime}\right)\right]^{2}}
$$

for the renormalized frequency of the atom. We note that the difficulty of solving the scalar equation (A3) does not scale with the number of bosonic modes under consideration. This fact deserves to be highlighted as the optimal value of $f$ is used to initialize the optimizer before executing the variational quantum algorithm presented in the main text and in more details in Appendix A 2. As discussed in
Appendix A 3, this is no longer true in the general case of $N>$ 1 , where the complexity of initialization scales exponentially with $N$

We can gain an understanding of how the polaron transformation works by analyzing the behavior of approximate solutions to Eq. (A3) at the boundaries of the range $1 \geqslant$ $\omega_{q}^{\prime} / \omega_{q} \geqslant 0$. Indeed, for small coupling strengths $g_{k}$, we expect $\omega_{q}^{\prime}$ to differ only slightly from $\omega_{q}$ and thus to be able to approximate $\omega_{q}^{\prime} / \omega_{q} \simeq 1-\epsilon$, where $\epsilon \ll 1$. In contrast, for large coupling strengths compared to the system frequencies, we expect $\omega_{q}^{\prime}$ to vanish exponentially and thus $\omega_{q}^{\prime} / \omega_{q} \simeq \epsilon$ to hold In the latter situation, the parameters of the polaron transformation approach the asymptotic scaling $f_{k} \simeq g_{k} / \omega_{k}$ leading to $g_{k}^{\prime} \rightarrow 0$ (along with $\omega_{q}^{\prime} \rightarrow 0$ ). This analysis indicates that the light-matter system effectively decouples in the polaron frame [see Eq. (A2)], both in the strong- and deep-strongcoupling regimes. As demonstrated below, the disentangling capabilities of this transformation and the solution of Eq. (A3) interpolate smoothly in the intermediate ultrastrong-coupling regime, making this tool suitable for investigating the lightmatter ground state in a very broad range of parameters.

We confirm the intuition developed in the above paragraph by analyzing both the single- and two-mode quantum Rabi Hamiltonians in resonance conditions $\omega_{q}=\omega_{k} \equiv \omega$, for $g_{k} \equiv$ $g \in[0, \omega]$. This is also the regime of parameters considered in the main text. Here, however, we are also interested in quantifying how well the state $P|\mathrm{vac}\rangle$ approximates the ground state of the multimode Rabi Hamiltonian in all coupling regimes. Figure 4(a) compares the expectation value $\left\langle H^{\prime}\right\rangle_{\text {vac }}$ to the exact ground-state energy of the single-mode (left) and twomode configurations (right). We observe that $\left\langle H^{\prime}\right\rangle_{\text {vac }}$ follows closely the exact solution in the range $g / \omega \in[0,2]$. This agreement is even improved for larger coupling strengths. Figures 4(b)-4(d) show the effective atom frequency $\omega_{q}^{\prime}$ and coupling strengths $g_{k}^{\prime}$ along with the $f_{k}$ parameters of the polaron transformation. Solid lines correspond to the numerical optimization of $\omega_{q}^{\prime}$, while dashed lines are obtained analytically from a series expansion of the right-hand side of Eq. (A3) to second order in the small parameter $\epsilon$ for both $\omega_{q}^{\prime} / \omega_{q} \simeq 1-\epsilon$ and $\omega_{q}^{\prime} / \omega_{q} \simeq \epsilon$ regimes. As anticipated, we observe an exponential reduction of the ratio $\omega_{q}^{\prime} / \omega_{q}$ along with the asymptotic tendency $f_{k} \simeq g_{k} / \omega_{k}$ and $g_{k}^{\prime} \rightarrow 0$ as $g$ increases. Although the energy of the state $P|\mathrm{vac}\rangle$ follows closely that of the ground state in the laboratory frame in the DSC regime, we note that $f_{k}$ grows linearly with $g_{k}$ in such conditions. This unfavorable scaling would make the Trotter-decomposition-based approach employed in the main text rather inefficient, requiring quantum circuits of larger depth to reach convergence.

The advantages of investigating the ground-state properties in the polaron frame are also emphasized by noticing that Eq. (A2) can be recast into

$$
\begin{aligned}
H_{f}^{\prime} / \hbar \simeq & \frac{\omega_{q}^{\prime}}{2} \sigma^{z}+\sum_{k=1}^{M} \omega_{k} a_{k}^{\dagger} a_{k}+2 \omega_{q}^{\prime} f_{k}\left(\sigma^{+} a_{k}+\sigma^{-} a_{k}^{\dagger}\right) \\
& -2 \omega_{q}^{\prime} \sigma^{z} \sum_{k, k^{\prime}=1}^{M} f_{k} f_{k^{\prime}} a_{k}^{\dagger} a_{k^{\prime}}-\sum_{k=1}^{M} \frac{g_{k}^{2}}{\omega_{k}+\omega_{q}^{\prime}}+\cdots,
\end{aligned}
$$


(a)

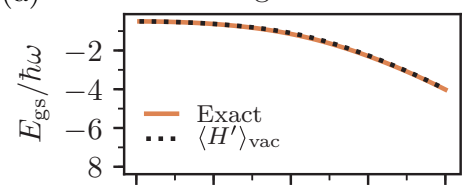

(b)

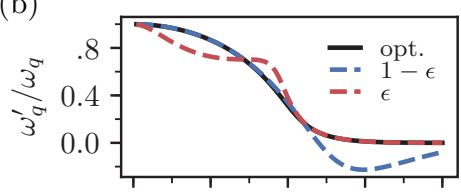

(c)

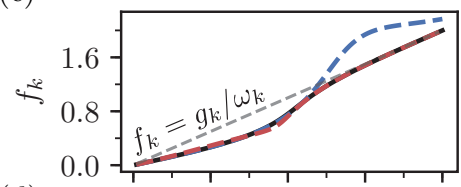

(d)
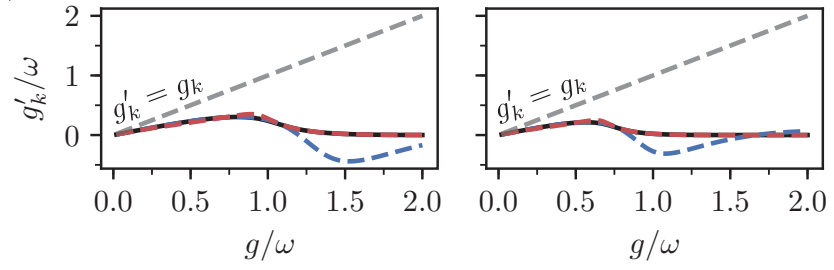

FIG. 4. Performance of polaron transformation in the strong-, ultrastrong-, and deep-strong-coupling regimes for the single- and two-mode quantum Rabi models in resonance conditions. (a) Comparison between $\left\langle H^{\prime}\right\rangle_{\text {vac }}$ and the exact ground-state energy. The present scale emphasizes the qualitative agreement between these two quantities, although it does not capture relatively small deviations which can be appreciated in the main text. (b)-(d) Show the polaron transformation parameters (black solid lines) obtained by numerical optimization. Dashed lines are analytical approximations of Eq. (A3) at the boundaries of $1 \geqslant \omega_{q}^{\prime} / \omega_{q} \geqslant 0$. The asymptotic regime of $f_{k} \simeq g_{k} / \omega_{k}$ is indicated in (c) (gray dashed lines). A similar line style in (d) indicates the coupling in the bare frame $g_{k}$. We observe that $g_{k}^{\prime}$ becomes exponentially small compared to $g_{k}$ as the bare coupling strength increases. This fact highlights the advantages of working in the polaron frame.

assuming $g_{k}^{\prime} /\left(\omega_{k}+\omega_{q}^{\prime}\right) \ll 1$ and expanding $q_{f}$ to first order. Equation (A4) is reminiscent of the multimode JaynesCummings model, with an additional term $\propto \sigma^{z} a_{k}^{\dagger} a_{k^{\prime}}$, that can either shift the frequency of the atom as a function of the number of photons in the $k$ modes $\left(k=k^{\prime}\right)$ or allow for the exchange of an excitation between two $k$ modes through the atom $\left(k \neq k^{\prime}\right)$. Provided that the effect of the latter interaction is only perturbative, the ground state of Eq. (A4) is the vacuum state. As shown in the main text, these conditions also lead to relatively small $f_{k}$ parameters, making it possible to construct a short-depth variational form by Trotter decomposition of the polaron transformation.

\section{Quantum-circuit compilation of the polaron variational form}

We now provide further details on the quantum-circuit compilation of the polaron variational form designed for the multimode quantum Rabi model. Specifically, we seek to (a)

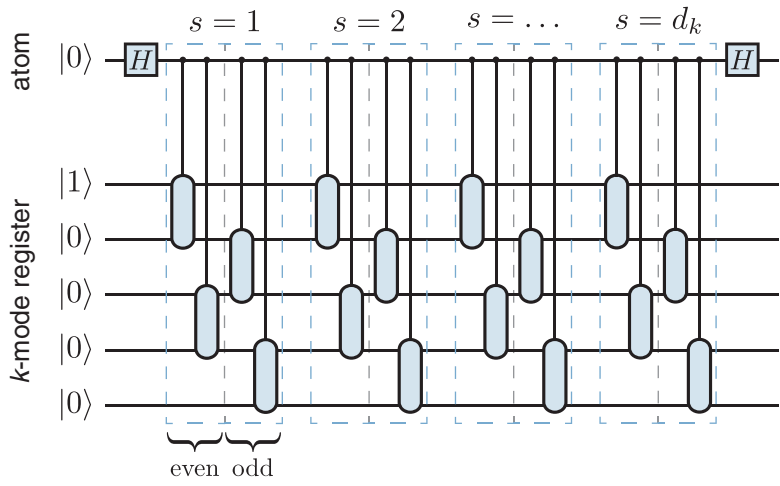

(b)

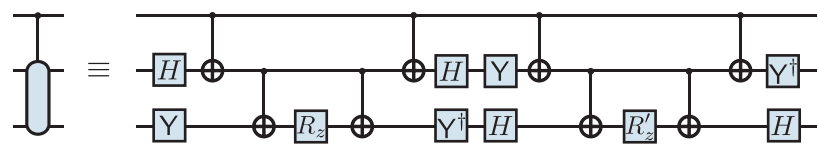

FIG. 5. Quantum-circuit compilation of the polaron variational form [Eq. (A5)] for one of the $k$-mode registers. (a) Schematic representation of the variational form as a sequence of controlled twoqubit gates. Such gates correspond to the unitaries $\exp \left(f_{k}^{s} \sigma^{x} \widetilde{X}_{k}^{e} / d_{k}\right)$ and $\exp \left(f_{k}^{s} \sigma^{x} \widetilde{X}_{k}^{o} / d_{k}\right)$, performed at each Trotter step labeled by $s$. (b) Compilation of the controlled two-qubit gate in one- and twoqubit gates available on the IBM quantum hardware [42]. Here, $H$ denotes the Hadamard gate, $Y=R_{x}(\pi / 2)$, and $R_{z}, R_{z}^{\prime}$ are rotations around the $Z$ axis using the conventional notation in which $R_{\mu}(\theta)=\exp \left(-i \theta \sigma^{\mu} / 2\right)$ for $\mu=x, y, z$. If the parameters $f_{k}^{s}$ are taken to be independent of the $k$-mode photon number, then $R_{z}=$ $R_{z}\left(f_{k}^{s} \sqrt{n_{k}+1} / d_{k}\right)$ and $R_{z}^{\prime}=R_{z}^{\dagger}$, where $n_{k}$ labels the sites within the $k$-mode register. If, in contrast, $f_{k}^{s} \rightarrow f_{k}^{s}\left(n_{k}\right)$ is allowed to vary from site to site, then $R_{z}=R_{z}\left[f_{k}^{s}\left(n_{k}\right) \sqrt{n_{k}+1} / d_{k}\right]$ and $R_{z}^{\prime}=R_{z}^{\dagger}$ or $R_{z}^{\prime}=R_{z}\left[f_{k}^{s^{\prime}}\left(n_{k}\right) \sqrt{n_{k}+1} / d_{k}\right]$ if one wishes to introduce an extra degree of freedom, $f_{k}^{s^{\prime}}\left(n_{k}\right)$, per controlled two-qubit gate. Importantly, the parameters of the variational form are initialized to the value specified by the polaron transformation in Eq. (4).

rewrite the unitary

$$
\text { Varform }=\prod_{k=1}^{M} \prod_{s=1}^{d_{k}} \exp \left(\frac{f_{k}^{s}}{d_{k}} \sigma^{x} \widetilde{X}_{k}^{e}\right) \exp \left(\frac{f_{k}^{s}}{d_{k}} \sigma^{x} \widetilde{X}_{k}^{o}\right)
$$

as a sequence of single- and two-qubit gates available on IBM's online quantum platform. The operator $\widetilde{X}_{k}^{e}$ in Eq. (A5), which was introduced in the main text, is defined as

$$
\tilde{X}_{k}^{e}=-i \sum_{n_{k} \text { even }}^{n_{k}^{\max }-1} \sqrt{n_{k}+1}\left(\sigma_{n_{k}}^{x} \sigma_{n_{k}+1}^{y}-\sigma_{n_{k}}^{y} \sigma_{n_{k}+1}^{x}\right) / 2 .
$$

$\widetilde{X}_{k}^{o}$ is defined analogously, with $n_{k}$ running over odd numbers in $\left[0, n_{k}^{\max }-1\right]$. Since the Pauli products in Eq. (A6) commute with each other, unitaries of the form $\exp \left(f_{k}^{s} \sigma^{x} \widetilde{X}_{k}^{e, o} / d_{k}\right)$ factorize into a sequence of controlled two-qubit gates that can be parallelized over the $k$-mode qubit registers. As shown in Fig. 5(a), these two-qubit gates operate on pairs of nextneighbor qubits of the $k$-mode registers and are controlled by the atom qubit. Figure 5(b) provides the compilation of the 
controlled two-qubit gates in elementary single- and two-qubit gates [42].

The atom and the $k$-mode registers are initialized to the states $|0\rangle$ and $\left|\widetilde{0}_{k}\right\rangle=|10 \ldots 0\rangle$, respectively, which correspond to the noninteracting ground states. This step is followed by $d_{k}$ sets of gates, where $d_{k}$ is the Trotter depth used to decompose the polaron unitary for mode $k$. In its simplest form, the polaron variational form has a single parameter per Trotter step. For a given $s$, the two-qubit-gate rotation angle is determined by such a parameter and the site index $n_{k}$ which introduces an additional scaling factor $\propto \sqrt{n_{k}}$ to this angle. The latter factor arises from the $\sqrt{n_{k}}$ scaling of the matrix elements of the harmonic-oscillator ladder operators $\left(a_{k}\right.$ and $\left.a_{k}^{\dagger}\right)$ in the photon-number basis. A version of the variational form with a larger number of parameters can easily be crafted by letting the two-qubit-gate rotation angle vary and potentially depart from the $\sqrt{n_{k}}$ scaling. Moreover, an additional parameter can be introduced if the controlled two-qubit gates are implemented as in Fig. 5(b), as this gate compilation uses two $R_{z}$ rotations that could be made independent from each other. In all cases, the initial value of the variational-form parameters must be set to that of the polaron transformation in Eq. (4).

An alternative option to the gate in Fig. 5(b) is the implementation a three-qubit gate at a hardware level without the need of gate compilation. This possibility, investigated in depth in Appendix E, has a number of advantages with respect to the compiled version of such gate. In fact, a hardware-level implementation of the controlled two-qubit gates would lead to a reduction of the gate count, potentially enabling the simulation of systems of larger size. Moreover, the gate generator can be engineered to conserve the number of excitations in the $k$-mode registers and thus the SES encoding. Since these criteria are not guaranteed by the complied version of the gate in Fig. 5(b), an error occurring halfway in such a sequence could severely impact the state fidelity.

\section{Polaron transformation and variational form for the multimode Dicke Hamiltonian}

In this section, we extend our approach to treat the multimode Dicke Hamiltonian [Eq. (2)]. To this end, the polaron transformation needs to be modified to include the $N$ atoms as

$$
P_{\left\{f_{i}\right\}}=\exp \left[\sum_{i=1}^{N} \sum_{k=1}^{M} \sigma_{i}^{x} f_{i k}\left(a_{k}-a_{k}^{\dagger}\right)\right],
$$

where the real parameters $f_{i k}$, organized in the vectors $f_{i}=$ $\left(f_{i 1}, \ldots, f_{i M}\right)$, for $i=1, \ldots, N$, now depend on both the atom and the $k$-mode indices [22]. By transforming the Hamiltonian in Eq. (2) as $H \rightarrow H_{\left\{f_{i}\right\}}^{\prime}=P_{\left\{f_{i}\right\}}^{\dagger} H P_{\left\{f_{i}\right\}}$, we find

$$
\begin{aligned}
H_{\left\{f_{i}\right\}}^{\prime} / \hbar= & \sum_{i=1}^{N} \frac{\omega_{q i}^{\prime}}{2} \sigma_{i}^{z} q_{-f_{i}}^{\dagger} q_{f_{i}}+\sum_{k=1}^{M} \omega_{k} a_{k}^{\dagger} a_{k} \\
& +\sum_{i=1}^{N} \sum_{k=1}^{M} g_{i k}^{\prime} \sigma_{i}^{x}\left(a_{k}+a_{k}^{\dagger}\right)+\sum_{i, i^{\prime}=1}^{N} J_{i i^{\prime}} \sigma_{i}^{x} \sigma_{i^{\prime}}^{x},
\end{aligned}
$$

which generalizes Eq. (A2). Here, we derive parameters analogous to those introduced for the quantum Rabi model, including renormalized atom frequencies $\omega_{q i}^{\prime}=\omega_{q i} e^{-2 f_{i} \cdot f_{i}}$ and light-matter coupling constants $g_{i k}^{\prime}=\left(g_{i k}-\omega_{k} f_{i k}\right)$, along with the set of operators $q_{f_{i}}=\exp \left(2 \sigma_{i}^{x} \sum_{k=1}^{M} f_{i k} a_{k}\right)$ for $i=$ $1, \ldots, N$. Unlike the $N=1$ case, Eq. (A8) also includes an effective two-body coupling $J_{i i^{\prime}}=\sum_{k=1}^{M}\left(\omega_{k} f_{i k} f_{i^{\prime} k}-2 g_{i k} f_{i^{\prime} k}\right)$ between two atoms $i$ and $i^{\prime}$, which is mediated by the $k$ modes.

Due to the latter interaction, the ground state of Eq. (A8) is not necessarily close to that of the noninteracting case. Instead, we assume a ground-state Ansatz of the form $\prod_{k=1}^{M}\left|\psi_{a}\right\rangle\left|\widetilde{0}_{k}\right\rangle$, where $\left|\psi_{a}\right\rangle$ is the ground state of the effective spin Hamiltonian

$$
H_{a} / \hbar=\sum_{i=1}^{N} \frac{\omega_{q i}^{\prime}}{2} \sigma_{i}^{z}+\sum_{i, i^{\prime}=1}^{N} J_{i i^{\prime}} \sigma_{i}^{x} \sigma_{i^{\prime}}^{x}
$$

By minimizing the energy of $H_{a}$, one finds a parametrization $\left\{\boldsymbol{f}_{i}\right\}$ of the polaron transformation, that approximately decouples the atoms from the $k$ modes in Eq. (A8) [22]. In contrast to the case of the quantum Rabi model, the cost of finding a proper disentangling transformation now scales exponentially with system size.

Focusing on the limit of small $N$, Eq. (A9) can still be handled on a classical processor which is used for diagonalizing $H_{a}$ and optimizing its ground-state energy as a function of $\left\{\boldsymbol{f}_{i}\right\}$. It is worth mentioning that quantum routines, such as variational eigensolvers and quantum annealing, might also be useful for this task. In particular, the latter method is attractive for large $N$ due to the possibility of embedding Ising-type Hamiltonians with long-range interactions into physical models with bounded connectivity.

Regardless of how the above optimization is performed, a quantum circuit is needed to prepare the state $\left|\psi_{a}\right\rangle$ on the atom registers. This initialization step is followed by the application of the polaron variational form

$$
\text { Varform }=\prod_{i=1}^{N} \prod_{k=1}^{M} \prod_{s=1}^{d_{i k}} \exp \left(\frac{f_{i k}^{s}}{d_{i k}} \sigma_{i}^{x} \widetilde{X}_{k}^{e}\right) \exp \left(\frac{f_{i k}^{s}}{d_{i k}} \sigma_{i}^{x} \widetilde{X}_{k}^{o}\right),
$$

where $\sigma_{i}^{x}$ is the Pauli- $X$ operator for the $i$ th atom, and $d_{i k}$ is the Trotter order of the polaron unitary involving this qubit and the $k$ mode labeled by $k$. Furthermore, $f_{i k}^{s}$ are the parameters of the polaron variational form, adding to a total of $\sum_{i=1}^{N} \sum_{k=1}^{M} d_{i k}$ parameters, which scales linearly with the number of atoms. Additional parameters can be introduced by allowing $f_{i k}^{s} \rightarrow f_{i k}^{s}\left(n_{k}\right)$ to depend on the $k$-mode photonnumber index.

Figure 6 shows a schematic of the variational form including the initialization step. We assume that $\left|\psi_{a}\right\rangle$ can be synthesized by a set of $d_{a}$ hardware-efficient layers acting on the atom registers [3]. Although this is not scalable to large $N$, the choice of a hardware-efficient approach is motivated by the lack of structure in Eq. (A9). As previously shown in Fig. 5, the polaron variational form contains sets of controlled two-qubit gates acting on the $k$-mode registers. In the present case, the control qubit is swept across the atom registers, while the number of Trotter steps $d_{i k}$ may vary from one set to the other. Finally, we note that the compilation of the controlled two-qubit gates in one- and two-qubit gates is the same as in Fig. 5(b). 


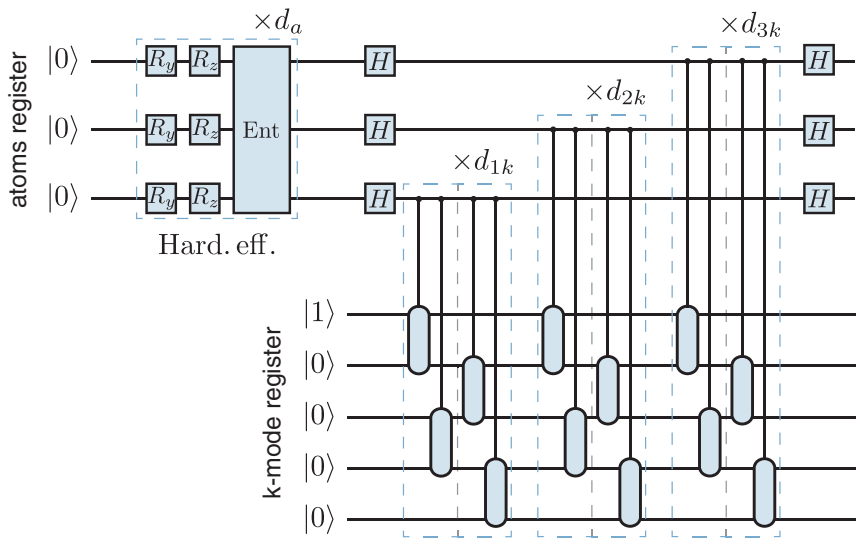

FIG. 6. Quantum-circuit compilation of the variational form for the multimode Dicke problem for three atoms and a single $k$-mode register. The variational form is initialized on the state $\prod_{k=1}^{M}\left|\psi_{a}\right\rangle\left|\widetilde{0}_{k}\right\rangle$ by means of hardware-efficient layers acting on the atom registers. The hardware-efficient variational form is followed by layer of controlled two-qubit gates identical to those in Fig. 5(b). Here, the control qubit is swept over the atom registers, and the number of Trotter steps may vary with the atom and $k$-mode indices.

\section{APPENDIX B: PERFORMANCE OF THE VQA ON A QUANTUM PROCESSOR}

\section{VQA simulations with different hardware-noise levels}

Mitigating the effect of noise is one of the greatest challenges for near-term quantum computers. In order to first quantify this effect, it is useful to investigate the performance of the VQA for a variable noise strength. One way to modify the effective level of noise that a quantum algorithm is subject to is to perform the quantum gates necessary for the computation having made these artificially slower. This enhances the effect of any decoherence channel and thus leads to an increased noise strength. Although we do not have low-level access to the pulses applied on the quantum hardware, like in Ref. [7], we can simulate the effect of a variable noise strength on the VQA by modifying the error model accordingly. This strategy also allows us to simulate a noise level below the calibrated values for the quantum device in use, which are provided by QISKIT [34].

We modify the noise level in simulation defining a noise factor $\eta_{\text {noise }}$, such that

$$
\begin{aligned}
T_{1} & =T_{1}^{\text {device }} / \eta_{\text {noise }}, \\
T_{2} & =T_{2}^{\text {device }} / \eta_{\text {noise }}, \\
r_{1 \mathrm{q}-\mathrm{g}} & =\eta_{\text {noise }} r_{1 \mathrm{q}-\mathrm{g}}^{\text {device }}, \\
r_{2 \mathrm{q}-\mathrm{g}} & =\eta_{\text {noise }} r_{2 \mathrm{q}-\mathrm{g}}^{\text {device }}, \\
r_{\text {readout }} & =\eta_{\text {noise }} r_{\text {readout }}^{\text {device }},
\end{aligned}
$$

where $T_{1}$ and $T_{2}$ are single-qubit relaxation and dephasing times, $r_{1 \mathrm{q}-\mathrm{g}}$ and $r_{2 \mathrm{q}-\mathrm{g}}$ are single- and two-qubit gate error probabilities, and $r_{\text {readout }}$ is the readout error probability, respectively. The quantities in Eq. (B1) which are labeled as "device" correspond to calibrated values for the day that the runs were executed.

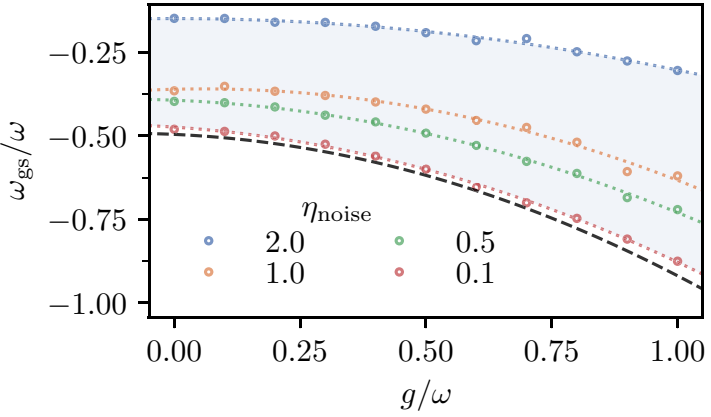

FIG. 7. Simulation of the VQA performance under variable noise strength. $\eta_{\text {noise }}=1$ corresponds to the realistic noise model provided by QISKIT for the IBM Q Poughkeepsie device. The shared area indicates the range of results which are possible with levels of noise $\eta_{\text {noise }}=0.1-2$. Extrapolation to zero noise indicates that results with acceptable accuracy are only attainable with levels of noise one order of magnitude smaller than those in the current generation of devices.

Figure 7 shows the result of the simulations with variable noise strength. We use the simultaneous-perturbationstochastic-approximation (SPSA) method as classical optimizer, with parameters $\alpha=0.602$ and $\gamma=0.101$ defined in Ref. [3]. We perform 25 calibration steps to compute the parameters $a$ and $c$ and another 100 SPSA trials for the actual optimization procedure. From the simulations, we conclude that VQA results with acceptable accuracy with respect to the exact ground-state energy would be attainable with noise levels that are one order of magnitude smaller than the actual ones. We note that extrapolation schemes to the zero-noise regime, like the ones discussed in Refs. [7,43], can potentially help to further mitigate the effects of noise.

\section{Simulations on the quantum processor}

For the quantum-hardware runs we use 3 qubits out of the 20 available on the IBM Q Poughkeepsie chip. The connectivity map of the device is shown in Fig. 8. The average error rates recorded throughout our experiments were $(5.25 \pm 0.212) \times 10^{-3}$ and $(3.75 \pm 0.364) \times 10^{-2}$ for singlequbit gates and controlled NOT (CNOT) gates, respectively. These error rates are highly dependent on the actual date on which the experiment took place. Mitigation of readout

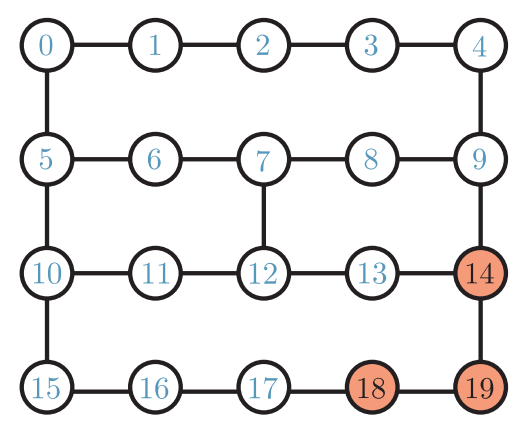

FIG. 8. Connectivity map of IBM Q Poughkeepsie quantum processor. Qubits 14, 18, and 19 (highlighted) were used for our experiment. The choice of qubits was dependent on the respective single- and two-qubit gate errors. 
errors is done with the standardized methods provided by QISKIT-IGNIS. There, a measurement calibration matrix is used to identify readout errors by preparing $2^{N}$ basis states and estimating the probability distribution of such state, with $N$ the number of qubits in simulation. The probability distribution of an unknown state can then be corrected based on these estimates. The calibration matrix was updated after every run or every 120 minutes of wall-clock time for the VQA runs.

Beyond coherent and incoherent errors on quantum hardware, the main limitation to greater accuracy has been found to be the classical optimizer. Indeed, the SPSA method fails to acquire the expected solution in several cases, potentially getting stuck into local minima. We confirm this hypothesis indirectly by performing the optimization of the variational Ansatz in simulation assuming the calibrated noise model for the device. We then compute the expectation value of such variational states on quantum hardware. This additional experiment can reach significantly better accuracy than those obtained by running the optimization over quantum-hardware energy estimations, as shown in Fig. 2. We therefore expect new and more powerful optimization algorithms to enable higher-accuracy VQA results.

\section{APPENDIX C: SAMPLING THE JOINT WIGNER FUNCTION}

We now provide details about the sampling of the joint Wigner distribution in Eq. (8). We begin by noticing that measurements of the Pauli products $\sigma_{1}^{l_{1}} \ldots \sigma_{N}^{l_{N}}$ can be done in the computational basis, provided that a set of single-qubit gates $\left\{R_{l_{i}}\right\}$ are executed on the atom register prior to qubit readout. Taking this into consideration, we now focus on the case where the Pauli string $\sigma_{1}^{l_{1}} \ldots \sigma_{N}^{l_{N}}$ contains only $\sigma^{z}$ and/or identity operators and no prior rotation of $\rho$ is needed.

Equation (8) makes use of the probability distribution of the displaced density matrix $\widetilde{D}^{\dagger}(\boldsymbol{\alpha}) \tilde{\rho} \widetilde{D}(\boldsymbol{\alpha})$. In order to sample such a distribution, $\widetilde{D}^{\dagger}(\boldsymbol{\alpha})$ needs first to be compiled into single- and two-qubit gates. Since this joint-displacement operator is a product of single-mode displacements of the form $\widetilde{D}^{\dagger}\left(\alpha_{k}\right)$, we only provide the quantum-circuit compilation for the latter unitary. To this end, it is convenient to introduce the real $\left(\alpha_{k}^{R}\right)$ and imaginary $\left(\alpha_{k}^{I}\right)$ parts of the displacement parameter $\alpha_{k}=\alpha_{k}^{R}+i \alpha_{k}^{I}$, and to expand the displacement operator as $\widetilde{D}^{\dagger}\left(\alpha_{k}\right) \simeq \exp \left[\alpha_{k}^{R}\left(\tilde{a}_{k}-\tilde{a}_{k}^{\dagger}\right)\right] \exp \left[-i \alpha_{k}^{I}\left(\tilde{a}_{k}+\tilde{a}_{k}^{\dagger}\right)\right]$, where " $\simeq$ " indicates an equivalence up to a global phase. Making use of the site operators of the $k$-mode registers, we find

$$
\begin{aligned}
& \tilde{a}_{k}-\tilde{a}_{k}^{\dagger}=-i \sum_{n_{k}=0}^{n_{k}^{\max }-1} \sqrt{n_{k}+1}\left(\sigma_{n_{k}}^{x} \sigma_{n_{k}+1}^{y}-\sigma_{n_{k}}^{y} \sigma_{n_{k}+1}^{x}\right) / 2, \\
& \tilde{a}_{k}+\tilde{a}_{k}^{\dagger}=\sum_{n_{k}=0}^{n_{k}^{\max }-1} \sqrt{n_{k}+1}\left(\sigma_{n_{k}}^{x} \sigma_{n_{k}+1}^{x}-\sigma_{n_{k}}^{y} \sigma_{n_{k}+1}^{y}\right) / 2 .
\end{aligned}
$$

Splitting the $k$-mode registers into even- and odd-index qubit subsets, the exponentiation of the operators in Eq. (C1) can be implemented by a Trotter expansion, as it was done for the polaron variational form.

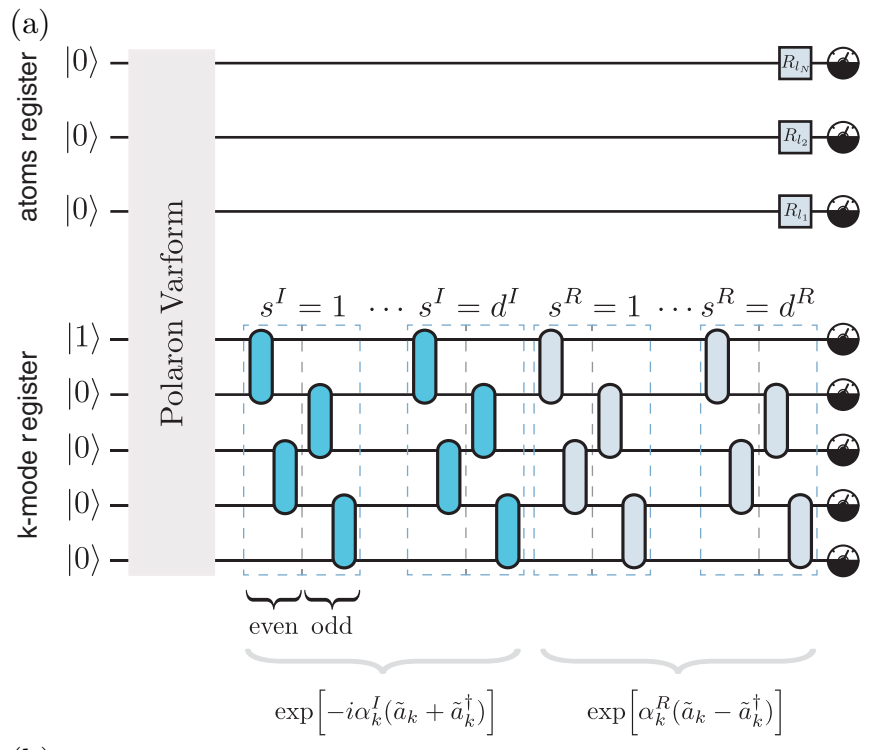

(b)

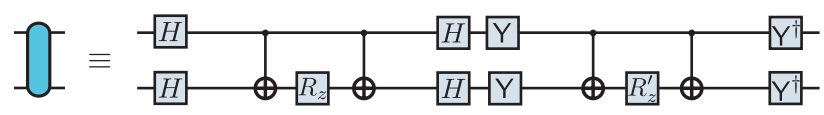

(c)

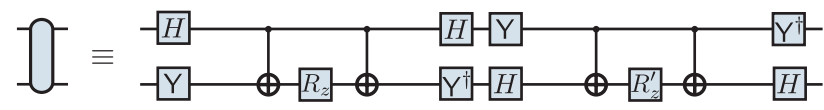

(d)

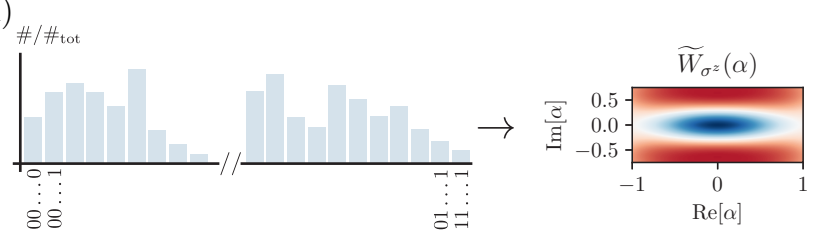

FIG. 9. Sampling the joint Wigner distribution corresponding to the atom and $k$-mode registers. (a) Quantum-circuit compilation of the displacement operators applied on the $k$-mode registers (here shown for a single mode), along with the single-qubit gates needed for tomography of the atom registers. These gates are executed on the ultrastrong-coupling ground state synthesized by the polaron variational form. The $k$-mode displacement unitary is split in real and imaginary components, implemented by gate sequences with Trotter depth $d^{R}$ and $d^{I}$, respectively, which may in general be different. The imaginary component is depicted first, and makes use of the two-qubit gates in (b) where $R_{z}=R_{z}\left(\alpha_{k}^{I} \sqrt{\tilde{n}_{k}+1} / d^{I}\right)$ and $R_{z}^{\prime}=R_{z}^{\dagger}$. What follows is the implementation of the real component of the displacement operator, which executes the two-qubit gates in (c) where $R_{z}=R_{z}\left(\alpha_{k}^{R} \sqrt{\tilde{n}_{k}+1} / d^{R}\right)$ and $R_{z}^{\prime}=R_{z}^{\dagger}$. Qubit readout is performed at the end of the quantum circuit in (a), leading to the histogram of counts in (d) after several repetitions of the experiment. This allows for reconstruction of the joint Wigner function as described in the main text.

Figure 9 summarizes the procedure for sampling the joint Wigner function with a set of tomography gates applied on the ultrastrong-coupling ground-state synthesized by the polaron variational form in Fig. 9(a). A first set of two-qubit gates, compiled in Fig. 9(b), implements a displacement operator along the imaginary- $\alpha_{k}$ axis with a Trotter order $d^{I}$. This is followed by a similar set of gates, compiled in Fig. 9(c), 
implementing a displacement along the real- $\alpha_{k}$ axis with Trotter depth $d^{R}$. Additionally, single-qubit tomography gates are applied on the atom registers. The circuit is terminated by readout of both the atom and $k$-mode registers. A histogram of counts [Fig. 9(d)] is constructed by repeating this procedure for a fixed $(\boldsymbol{l}, \boldsymbol{\alpha})$ pair. The joint Wigner function can then be computed from this histogram approximating the trace operator in Eq. (8) by

$$
\operatorname{Tr}_{q}[\ldots] \simeq \frac{2^{M}}{\pi^{M}} \sum_{q}(-1)^{\sum_{i=1}^{N} \beta_{q_{i}}} c\left(q_{1}, \ldots, q_{N} ; \tilde{n}_{1}, \ldots, \tilde{n}_{M}\right),
$$

where $c\left(q_{1}, \ldots, q_{N} ; \tilde{n}_{1}, \ldots, \tilde{n}_{M}\right)$ are the normalized counts for the basis vector $\left|q_{1}, \ldots, q_{N} ; \tilde{n}_{1}, \ldots, \tilde{n}_{M}\right\rangle$ in which $q_{i} \in$ $[0,1]$ is the state of the $i$ th atom, and $\beta_{q_{i}} \in[0,1]$ accounts for the presence of a $\sigma_{i}^{z}$ operator before qubit readout. More precisely, such a parameter is set according to the rules $\beta_{q_{i}}=1-q_{i}$ if $l_{i}=z$, and $\beta_{q_{i}}=0$ if $l_{i}=0$. We note that the approximate relation in Eq. (C2) can be replaced by an exact equivalence in the limit of large counts.

The reconstruction error scales with the amplitude of the displacement parameters $\left|\alpha_{k}\right|^{2}$, although it can be reduced by increasing the Trotter order in the implementation of the unitaries $\widetilde{D}^{\dagger}\left(\alpha_{k}\right)$. The finite Fock-state truncation of the encoded bosonic modes sets an upper bound to the accuracy of the reconstructed joint Wigner distribution, as demonstrated in Fig. 3. Given that the quantum circuit corresponding to such operators is appended to that of the polaron variational form, this procedure would ultimately be limited by the strength of the noise in the quantum processor. However, we find that Trotter depths as small as 2 are enough to demonstrate the qualitative features of the joint Wigner distribution.

\section{APPENDIX D: EFFECT OF PHASE- AND BIT-FLIP NOISE CHANNELS UNDER A SES ENCODING}

This Appendix discuses the so-called memory error of a small-qubit register encoding a bosonic mode. We consider both phase- and bit-flip error channels acting on a copy of the maximally entangled state $|\psi\rangle \sim \sum_{n_{k}=0}^{n_{k}^{\max }}\left|n_{k}\right\rangle$, in absence of logical gates. Specifically, we compute the state fidelity $F\left(\rho, \rho^{\prime}\right)=\operatorname{Tr}\left[\sqrt{\rho} \rho^{\prime} \sqrt{\rho}\right][44]$, where $\rho=|\psi\rangle\langle\psi| \otimes$ $|\psi\rangle\langle\psi|$ and $\rho^{\prime} \sim \sum_{n_{k}, n_{k}^{\prime}=0}^{n_{\max }}\left|n_{k}\right\rangle\left\langle n_{k}^{\prime}\right| \otimes \mathcal{E}_{\mathrm{C}}\left(\left|n_{k}\right\rangle\left\langle n_{k}^{\prime}\right|\right)$. Here, $\mathcal{E}_{\mathrm{C}}$ are multiqubit error channels obtained by composition of single-qubit ones, $\mathcal{E}_{q, \mathrm{C}}$. The latter have the general form $\mathcal{E}_{q, \mathrm{C}}(\bullet)=\sum_{i=0}^{1} E_{i} \bullet E_{i}^{\dagger}$, where $\left\{E_{i}\right\}$ are the Kraus operator for the channel $\mathrm{C}$. Denoting the error probability with $r$, we define the phase-flip channel by $E_{0}=\sqrt{1-r} \mathbb{1}, E_{1}=\sqrt{r} \sigma^{z}$, while $E_{0}=\sqrt{1-r} \mathbb{1}, E_{1}=\sqrt{r} \sigma^{x}$ correspond to the bit-flip channel.

Figure 10 shows the result for the state fidelity assuming a $k$-mode register containing up to a maximum of seven qubits. As anticipated in the main text, we find that bit-flip errors are the most relevant as the size of the $k$-mode register is increased. This can be understood intuitively by looking at the complement $\overline{\mathrm{SES}}$ of the SES subspace used for the encoding. Since the number of basis vectors in $\overline{\mathrm{SES}}$, and thus the dimension of this subspace, grows exponentially with $n_{k}^{\max }$, a noise operator breaking the SES symmetry could significantly (a) Phase flip

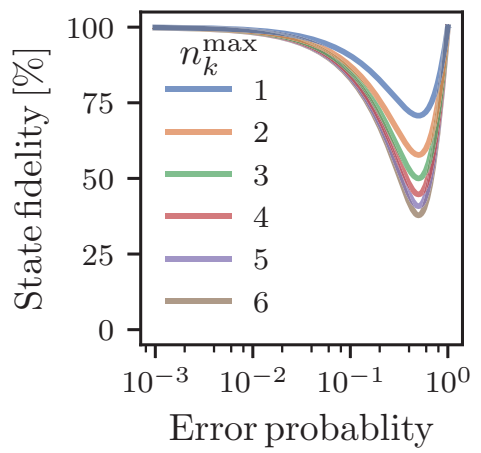

(b) Bit flip

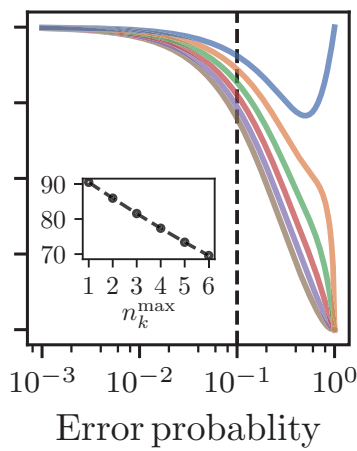

FIG. 10. Effect of phase-flip (a) and bit-flip (b) error channels on the fidelity of a maximally entangled state as a function of the total error probability. We consider a $k$-mode qubit register of size $n_{k}^{\max }+$ $1 \leqslant 7$. The legend applies for both left and right plots. We observe a significant decrease of the state fidelity as the size of the qubit register is increased. As discussed in the main text, bit-flip errors are expected to be dominant as the register size is scaled up to simulate a larger number of modes with also greater Fock-state truncation. The inset shows the state fidelity as a function of $n_{k}^{\max }$ for an error probability of $10^{-1}$.

affect the state fidelity in the limit of large $n_{k}^{\max }$. On the other hand, it is worth noticing that this might not necessarily limit the performance of near-term algorithms requiring only a small number of qubits. Alternatives for scaling up to larger devices include the use of qubits with naturally long $T_{1}$ times, or a different encoding for the bosonic modes [32,33]. Future work will investigate the performance crossover of the various possible encodings as the variational circuit is scaled up. Finally, we note that state fidelity, although standard, is a strong metric to evaluate the performance of our variational algorithm, and provides only a qualitative estimation of the impact on the energy of the variational Ansatz.

\section{APPENDIX E: CIRCUIT-QED IMPLEMENTATION OF THE CONTROLLED-EXCHANGE GATES}

With the purpose of reducing the gate count of the polaron variational form, we now present a superconducting-qubit implementation of a controlled-exchange gate. We stress, however, that the proposed approach could be leveraged by any other quantum-hardware platform with native interactions similar to those found in a standard circuit-QED setup. Below, we provide an ideal implementation of the gate interaction and then suggest a superconducting circuit that approaches the ideal scheme.

We first consider the case of a single atom and $k$-mode registers. The frequency of the physical qubit corresponding to the atom is denoted by $\omega_{\nu}$, while the frequencies of the qubits belonging to the $k$-mode register are denoted by $\omega_{n_{k}}$, with $n_{k} \in\left[0,1, \ldots, n_{k}^{\max }\right]$. Note that these frequencies are not related to the parameters of the problem that one wishes to simulate. With the purpose of engineering a controlled twoqubit gate, we assume the two qubits of the $k$-mode register, labeled by $\mu \in\left[n_{k}, n_{k}+1\right]$, to be independently coupled to the atom qubit with a time-dependent interaction strength. 
This situation is described by a three-qubit Hamiltonian of the form

$$
H_{\text {ideal }} / \hbar=\frac{\omega_{v}}{2} \sigma_{\nu}^{z}+\sum_{\mu}\left[\frac{\omega_{\mu}}{2} \sigma_{\mu}^{z}+\Omega_{\mu}(t)\left(\sigma_{\mu}^{+} \sigma_{\nu}^{-}+\sigma_{\mu}^{-} \sigma_{\nu}^{+}\right)\right],
$$

where we take, in particular, $\Omega_{\mu}=\Omega_{\mu}^{0}+2 \varepsilon_{\mu} \sin \left[\left(\omega_{\mu}-\omega_{\nu}+\right.\right.$ $\left.\delta_{\mu}\right) t+\phi_{\mu}$ ]. Here, $\Omega_{\mu}^{0}$ is an always-on interaction strength, $2 \varepsilon_{\mu}$ is the modulation amplitude, $\delta_{\mu}$ is a frequency detuning with respect to the $\mu-v$ transition, and $\phi_{\mu}$ is a relative phase. Counter-rotating terms of the form $\sigma_{\mu}^{+} \sigma_{v}^{+}$and its Hermitian conjugate have been omitted after a rotating-wave approximation (RWA).

To make the three-qubit-gate interaction explicit, we now perform a standard time-dependent Schrieffer-Wolff transformation with generator

$$
S=\sum_{\mu}\left[\frac{\Omega_{\mu}^{0}}{\omega_{\mu}-\omega_{\nu}}+\frac{i \varepsilon_{\mu} e^{-i\left[\left(\omega_{\mu}-\omega_{\nu}+\delta_{\mu}\right) t+\phi_{\mu}\right]}}{2\left(\omega_{\mu}-\omega_{\nu}\right)+\delta_{\mu}}\right] \sigma_{\mu}^{+} \sigma_{\nu}^{-}-\text {H.c. },
$$

conceived to remove first-order interaction terms by the condition $\left[H_{0}, S\right]+H_{\text {int }}-i \dot{S}=0$, where $H_{0}=\frac{\omega_{v}}{2} \sigma_{v}^{z}+$ $\sum_{\mu} \frac{\omega_{\mu}}{2} \sigma_{\mu}^{z}$ and $H_{\text {int }}=\sum_{\mu} \Omega_{\mu}(t)\left(\sigma_{\mu}^{+} \sigma_{\nu}^{-}+\sigma_{\mu}^{-} \sigma_{\nu}^{+}\right)$. Assuming $\Omega_{\mu}^{0} /\left(\omega_{\mu}-\omega_{\nu}\right) \ll 1$ (dispersive regime) and $\varepsilon_{\mu} /\left[2\left(\omega_{\mu}-\right.\right.$ $\left.\left.\omega_{v}\right)+\delta_{\mu}\right] \ll 1$, we expand the transformed Hamiltonian up to second order in the interaction strength, and move to a frame rotating at frequencies $\omega_{\nu}$ for the atom qubit and $\omega_{\mu}+\delta_{\mu}$ for the $k$-mode qubits, where the modulated interaction is resonant. Setting the phase of the drives as $\phi_{n_{k}}=0$ and $\phi_{n_{k}+1}=-\pi / 2$, and performing a second RWA, we find the effective Hamiltonian

$$
H_{\text {ideal }}^{\prime} / \hbar=\frac{\xi_{n_{k}}}{2} \sigma_{\mu}^{z}\left(\sigma_{n_{k}}^{x} \sigma_{n_{k}+1}^{y}-\sigma_{n_{k}}^{y} \sigma_{n_{k}+1}^{x}\right)+\frac{\delta \omega_{v}}{2} \sigma_{v}^{z},
$$

where drive parameters have been chosen to satisfy $-\delta_{\mu}+$ $\left(\Omega_{\mu}^{0}\right)^{2} /\left(\omega_{\mu}-\omega_{v}\right)+\varepsilon_{\mu}^{2} / \delta_{\mu}^{\prime}=0$, with $\delta_{\mu}^{\prime}=2\left(\omega_{\mu}-\omega_{\nu}\right)+\delta_{\mu}$. The drive condition removes terms $\propto \sigma_{\mu}^{z}$ from Eq. (E3) and makes the three-qubit interaction resonant in the current frame. Moreover, $\xi_{n_{k}}$ has been defined as an effective exchange-interaction rate between the two neighboring qubits of the $k$-mode register, that is mediated by the atom qubit and given by

$$
\xi_{n_{k}}=\frac{1}{2} \frac{\varepsilon_{n_{k}} \varepsilon_{n_{k}+1}}{\delta_{n_{k}}^{\prime} \delta_{n_{k}+1}^{\prime}}\left(\delta_{n_{k}}^{\prime}+\delta_{n_{k}+1}^{\prime}\right) .
$$

Additionally, we derive a shift to the frequency of the atom qubit given by $\delta \omega_{\nu}=-\left(\Omega_{\mu}^{0}\right)^{2} /\left(\omega_{\mu}-\omega_{\nu}\right)-\varepsilon_{\mu}^{2} / \delta_{\mu}^{\prime}$, due to the interaction with the two other qubits and the presence of the drive.

Evolution under the Hamiltonian in Eq. (E3) generates the desired controlled-exchange gate operation $\exp \left[-i\left(\xi_{n_{k}} t / 2\right) \sigma_{\mu}^{z}\left(\sigma_{n_{k}}^{x} \sigma_{n_{k}+1}^{y}-\sigma_{n_{k}}^{y} \sigma_{n_{k}+1}^{x}\right)\right]$ which is key to the polaron variational form. Due to the presence of the term $\propto \delta \omega_{v}$ in Eq. (E3), an unintentional $R_{z}$ rotation on the atom qubit needs to be corrected for by applying an additional single-qubit gate. Modulation amplitudes $\varepsilon_{\mu} / 2 \pi$ of the order of $10 \mathrm{MHz}$ and typical values of $\delta_{\mu}^{\prime} / 2 \pi$ of the order of the $\mathrm{GHz}$ lead to controlled-exchange rates $\xi_{n_{k}} / 2 \pi$ in the range $0.1-0.5 \mathrm{MHz}$. Despite this number being small compared to standard rates of one- and two-qubit gates

in superconducting-qubit architectures, counting with a direct implementation of the three-qubit gate still provides a significant advantage with respect to its compiled counterpart in Fig. 5. In fact, the proposed gate is designed to conserve the excitation number of the $k$-mode registers and thus the SES encoding. Furthermore, while the gate time of the direct implementation is proportional to the desired rotation angle, the compiled version of the gate has an approximately fixed gate time determined by the number of CNOT gates in the circuit. This important difference would be leveraged further as the Trotter order of the polaron variational form is increased, making the controlled rotations closer to the identity.

Having presented an ideal model for the controlledexchange gate, we now elaborate on a possible superconducting-circuit implementation of the Hamiltonian

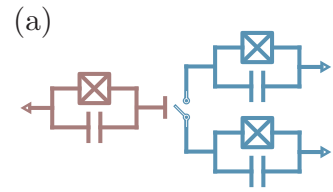

(c)
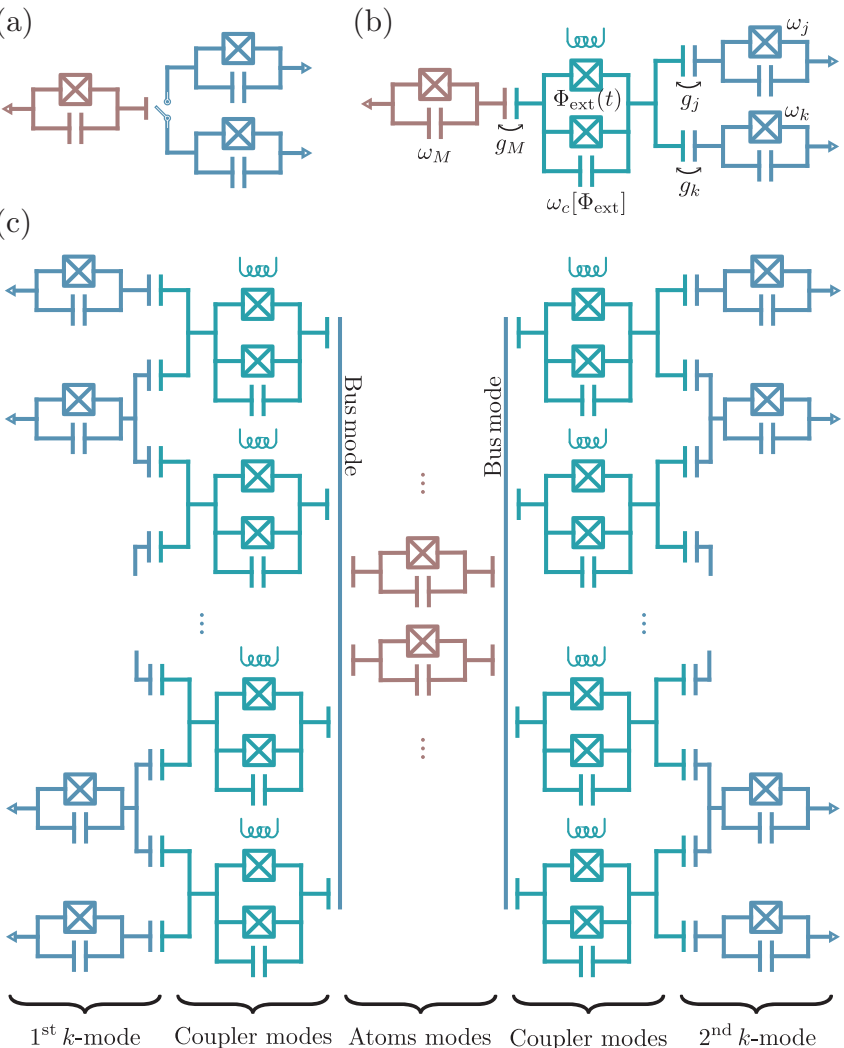

FIG. 11. Variational quantum-optics (VQO) superconducting processor. (a) Schematic of a controlled-exchange gate between three transmon qubits. The qubit in red plays the role of the atom register, controlling the switch on and off of an exchange interaction between two neighboring transmons belonging to the $k$-mode register. (b) Superconducting-qubit implementation of the concept in (a). A tunable coupler (light green) is introduced to mediate the interaction between the atom and $k$-mode registers. (c) Device for the VQO simulation of the ultrastrong interaction between $N$ atoms (in red) and two bosonic modes (light blue, left and right). A superconducting resonator acting as a quantum bus is required to enable long-range interactions between the atoms and the coupler modes. Moreover, the bus mode enables dispersive two-qubit gates between the atom qubits if made frequency tunable [46], which are required for state preparation in the case of $N>1$. 
in Eq. (E1). In particular, we consider an architecture made of transmon qubits and tunable couplers (see Fig. 11), similar to that studied in Ref. [45]. Using couplers to mediate parametric interactions allows us to remove the need for frequency tunability of the qubit modes resulting in greater coherence times. Standard circuit quantization of the unit-cell device in Fig. 11(b), followed by a two-level and rotating-wave approximations, leads to the Hamiltonian

$$
\begin{aligned}
H= & \frac{\omega_{c}^{\mathrm{b}}\left[\Phi_{\mathrm{ext}}\right]}{2} \sigma_{c}^{z}+\frac{\omega_{v}^{\mathrm{b}}}{2} \sigma_{v}^{z}+\sum_{\mu} \frac{\omega_{\mu}^{\mathrm{b}}}{2} \sigma_{\mu}^{z}+g_{v}\left(\sigma_{\mu}^{+} \sigma_{c}^{-}+\sigma_{\mu}^{-} \sigma_{c}^{+}\right) \\
& +\sum_{\mu} g_{\mu}\left(\sigma_{\mu}^{+} \sigma_{c}^{-}+\sigma_{\mu}^{-} \sigma_{c}^{+}\right),
\end{aligned}
$$

where $\omega_{c}^{\mathrm{b}}\left[\Phi_{\text {ext }}\right]$ denotes the bare frequency of the tunable coupler, $\omega_{v}^{\mathrm{b}}$ is the bare frequency of the atom qubit, $\left\{\omega_{\mu}^{\mathrm{b}}\right\}$ are the bare frequencies of two neighboring qubits in the $k$-mode register, and $\left\{g_{\nu}, g_{\mu}\right\}$ are the respective coupling strengths between such qubits and the coupler. We assume that the coupler frequency can be tuned and modulated by a external magnetic flux $\Phi_{\text {ext }}$ through the coupler's SQUID loop.

Following [45], we perform the adiabatic elimination of the coupler mode by means of a Schrieffer-Wolff transformation in the dispersive regime $g_{v} \ll \Delta_{v}^{\mathrm{b}}\left[\Phi_{\text {ext }}\right], g_{\mu} \ll \Delta_{\mu}^{\mathrm{b}}\left[\Phi_{\text {ext }}\right]$, where $\Delta_{\beta}^{\mathrm{b}}\left[\Phi_{\text {ext }}\right]=\omega_{\beta}^{\mathrm{b}}-\omega_{c}^{\mathrm{b}}\left[\Phi_{\text {ext }}\right]$. Assuming that the coupler mode remains in its ground state at all times, we derive an effective Hamiltonian of the form $H_{\text {eff }}=H_{\text {ideal }}+H_{\text {err }}$, where $H_{\text {ideal }}$ is the ideal interaction model given in Eq. (E1) with frequency parameters $\omega_{v}=\omega_{v}^{\mathrm{b}}+g_{v}^{2} / \Delta_{v}^{\mathrm{b}}\left[\Phi_{\text {ext }}\right], \omega_{\mu}=\omega_{\mu}^{\mathrm{b}}+$ $g_{\mu}^{2} / \Delta_{\mu}^{\mathrm{b}}\left[\Phi_{\text {ext }}\right]$ and flux-tunable coupling strengths

$$
\Omega_{\mu}\left[\Phi_{\mathrm{ext}}(t)\right]=\frac{g_{\mu} g_{v}}{2} \frac{\left(\Delta_{\mu}^{\mathrm{b}}\left[\Phi_{\mathrm{ext}}\right]+\Delta_{v}^{\mathrm{b}}\left[\Phi_{\mathrm{ext}}\right]\right)}{\Delta_{\mu}^{\mathrm{b}}\left[\Phi_{\mathrm{ext}}\right] \Delta_{v}^{\mathrm{b}}\left[\Phi_{\mathrm{ext}}\right]} .
$$

$H_{\text {err }}$ is a spurious off-resonant term coupling directly the two qubits of the $k$-mode register. Implementation of the interac- tion model in Eq. (E3) from $H_{\text {eff }}$ requires a two-tone modulation of $\Phi_{\text {ext }}(t)$ at frequencies $\omega_{\mu}-\omega_{v}+\delta_{\mu}$ for $\mu \in\left[n_{k}, n_{k}+\right.$ 1]. We observe that the effect of $H_{\text {err }}$ can be exactly canceled by tuning the qubits to the destructive-interference condition $\Delta_{n_{k}}^{\mathrm{b}}\left[\Phi_{\text {ext }}\right]=-\Delta_{n_{k}+1}^{\mathrm{b}}\left[\Phi_{\text {ext }}\right]$. However, this also leads to a small interaction strength for the controlled-exchange operation. A better alternative is to consider the two $k$-mode qubits being coupled to an additional ancillary mode whose frequency is chosen to counteract the effect of $H_{\mathrm{err}}$. Moreover, if the $k$-mode qubits are properly detuned the residual interaction only leads to a frequency renormalization of the drive condition above and to an off-resonant controlled-exchange interaction between $v$ and $n_{k}\left(n_{k}+1\right)$ via $n_{k}+1\left(n_{k}\right)$ which can be dropped by means of a RWA. As anticipated, we find that for typical circuit parameters and without optimization, the gate-interaction rate $\xi_{n_{k}}$ can reach values in the range of $0.1-0.5 \mathrm{MHz}$, assuming a modulation amplitude between $25 \%-50 \%$ of $\Omega_{\mu}^{0}$ [45]. Further improvements on the speed of the gate might be enabled by optimization of the proposed circuit, the use of other possible coupling schemes implementing Eq. (E3), or optimal control techniques [47].

The proposed implementation may be scaled up to a larger number of qubits, as shown schematically in Fig. 11(c) for the case of $N$ atoms and two $k$ modes. A cavity bus mode is used to enable long-range interactions between the tunable couplers and the qubits playing the role of atom registers. Moreover, the bus mode allows for the implementation of two-qubit gates between the atom qubits, which are necessary to initialize the polaron variational form for $N>1$. We note that the controlled-exchange gates can be parallelized over even and odd qubits of the $k$-mode registers. Finally, we stress that scaling up to a larger number of qubits entails issues that are beyond the scope of this work and requires to be examined in greater detail. The analysis of this Appendix, however, provides a path forward toward the implementation of variational quantum-optics algorithms on special-purpose hardware.
[1] A. Peruzzo, J. McClean, P. Shadbolt, M.-H. Yung, X.-Q. Zhou, P. J. Love, A. Aspuru-Guzik, and J. L. O'brien, A variational eigenvalue solver on a photonic quantum processor, Nat. Commun. 5, 4213 (2014).

[2] P. J. J. O’Malley, R. Babbush, I. D. Kivlichan, J. Romero, J. R. McClean, R. Barends, J. Kelly, P. Roushan, A. Tranter, N. Ding, B. Campbell, Y. Chen, Z. Chen, B. Chiaro, A. Dunsworth, A. G. Fowler, E. Jeffrey, E. Lucero, A. Megrant, J. Y. Mutus et al., Scalable Quantum Simulation of Molecular Energies, Phys. Rev. X 6, 031007 (2016).

[3] A. Kandala, A. Mezzacapo, K. Temme, M. Takita, M. Brink, J. M. Chow, and J. M. Gambetta, Hardware-efficient variational quantum eigensolver for small molecules and quantum magnets, Nature (London) 549, 242 (2017).

[4] J. I. Colless, V. V. Ramasesh, D. Dahlen, M. S. Blok, M. E. Kimchi-Schwartz, J. R. McClean, J. Carter, W. A. de Jong, and I. Siddiqi, Computation of Molecular Spectra on a Quantum Processor with an Error-Resilient Algorithm, Phys. Rev. X 8, 011021 (2018).
[5] C. Hempel, C. Maier, J. Romero, J. McClean, T. Monz, H. Shen, P. Jurcevic, B. P. Lanyon, P. Love, R. Babbush et al., Quantum Chemistry Calculations on a Trapped-Ion Quantum Simulator, Phys. Rev. X 8, 031022 (2018).

[6] J. R. McClean, J. Romero, R. Babbush, and A. Aspuru-Guzik, The theory of variational hybrid quantum-classical algorithms, New J. Phys. 18, 023023 (2016).

[7] A. Kandala, K. Temme, A. D. Córcoles, A. Mezzacapo, J. M. Chow, and J. M. Gambetta, Error mitigation extends the computational reach of a noisy quantum processor, Nature (London) 567, 491 (2019).

[8] N. Moll, P. Barkoutsos, L. S. Bishop, J. M. Chow, A. Cross, D. J. Egger, S. Filipp, A. Fuhrer, J. M. Gambetta, M. Ganzhorn et al., Quantum optimization using variational algorithms on near-term quantum devices, Quantum Sci. Technol. 3, 030503 (2018).

[9] J.-M. Reiner, M. Marthaler, J. Braumüller, M. Weides, and G. Schön, Emulating the one-dimensional fermi-hubbard model by a double chain of qubits, Phys. Rev. A 94, 032338 (2016). 
[10] E. F. Dumitrescu, A. J. McCaskey, G. Hagen, G. R. Jansen, T. D. Morris, T. Papenbrock, R. C. Pooser, D. J. Dean, and P. Lougovski, Cloud Quantum Computing of an Atomic Nucleus, Phys. Rev. Lett. 120, 210501 (2018).

[11] C. Ciuti and I. Carusotto, Input-output theory of cavities in the ultrastrong coupling regime: The case of time-independent cavity parameters, Phys. Rev. A 74, 033811 (2006).

[12] S. Ashhab and F. Nori, Qubit-oscillator systems in the ultrastrong-coupling regime and their potential for preparing nonclassical states, Phys. Rev. A 81, 042311 (2010).

[13] F. Beaudoin, J. M. Gambetta, and A. Blais, Dissipation and ultrastrong coupling in circuit QED, Phys. Rev. A 84, 043832 (2011).

[14] A. F. Kockum, A. Miranowicz, S. De Liberato, S. Savasta, and F. Nori, Ultrastrong coupling between light and matter, Nat. Rev. Phys. 1, 19 (2019).

[15] P. Forn-Díaz, L. Lamata, E. Rico, J. Kono, and E. Solano, Ultrastrong coupling regimes of light-matter interaction, Rev. Mod. Phys. 91, 025005 (2019).

[16] S. Haroche and J.-M. Raimond, Exploring the Quantum: Atoms, Cavities, and Photons (Oxford University Press, Oxford, 2006).

[17] A. Blais, R.-S. Huang, A. Wallraff, S. M. Girvin, and R. J. Schoelkopf, Cavity quantum electrodynamics for superconducting electrical circuits: An architecture for quantum computation, Phys. Rev. A 69, 062320 (2004).

[18] J. Bourassa, J. M. Gambetta, A. A. Abdumalikov Jr, O. Astafiev, Y. Nakamura, and A. Blais, Ultrastrong coupling regime of cavity qed with phase-biased flux qubits, Phys. Rev. A 80, 032109 (2009).

[19] D. Ballester, G. Romero, J. J. García-Ripoll, F. Deppe, and E. Solano, Quantum Simulation of the Ultrastrong-Coupling Dynamics in Circuit Quantum Electrodynamics, Phys. Rev. X 2, 021007 (2012).

[20] D. Braak, Integrability of the Rabi Model, Phys. Rev. Lett. 107, 100401 (2011).

[21] J. Hausinger and M. Grifoni, Qubit-oscillator system: An analytical treatment of the ultrastrong coupling regime, Phys. Rev. A 82, 062320 (2010).

[22] G. Dìaz-Camacho, A. Bermudez, and J. J. García-Ripoll, Dynamical polaron ansatz: A theoretical tool for the ultrastrongcoupling regime of circuit qed, Phys. Rev. A 93, 043843 (2016).

[23] T. Shi, Y. Chang, and J. J. García-Ripoll, Ultrastrong Coupling Few-Photon Scattering Theory, Phys. Rev. Lett. 120, 153602 (2018).

[24] N. Rivera, J. Flick, and P. Narang, Variational Theory of Nonrelativistic Quantum Electrodynamics, Phys. Rev. Lett. 122, 193603 (2019).

[25] J. Braumüller, M. Marthaler, A. Schneider, A. Stehli, H. Rotzinger, M. Weides, and A. V. Ustinov, Analog quantum simulation of the rabi model in the ultra-strong coupling regime, Nat. Commun. 8, 779 (2017).

[26] A. L. Grimsmo and S. Parkins, Cavity-qed simulation of qubitoscillator dynamics in the ultrastrong-coupling regime, Phys. Rev. A 87, 033814 (2013).

[27] A. C. Y. Li, A. Macridin, and P. Spentzouris (unpublished).

[28] R. Somma, G. Ortiz, E. Knill, and J. Gubernatis, Quantum simulations of physics problems, Int. J. Quantum. Inf. 1, 189 (2003).

[29] M. R. Geller, J. M. Martinis, A. T. Sornborger, P. C. Stancil, E. J. Pritchett, H. You, and A. Galiautdinov, Universal quantum simulation with prethreshold superconducting qubits: Singleexcitation subspace method, Phys. Rev. A 91, 062309 (2015).

[30] P. K. Barkoutsos, J. F. Gonthier, I. Sokolov, N. Moll, G. Salis, A. Fuhrer, M. Ganzhorn, D. J. Egger, M. Troyer, A. Mezzacapo et al., Quantum algorithms for electronic structure calculations: Particle-hole hamiltonian and optimized wave-function expansions, Phys. Rev. A 98, 022322 (2018).

[31] M. Avalle and A. Serafini, Noisy Quantum Cellular Automata for Quantum Versus Classical Excitation Transfer, Phys. Rev. Lett. 112, 170403 (2014).

[32] A. Macridin, P. Spentzouris, J. Amundson, and R. Harnik, Digital quantum computation of fermion-boson interacting systems, Phys. Rev. A 98, 042312 (2018).

[33] A. Macridin, P. Spentzouris, J. Amundson, and R. Harnik, Electron-Phonon Systems on a Universal Quantum Computer, Phys. Rev. Lett. 121, 110504 (2018).

[34] G. Aleksandrowicz, T. Alexander, P. Barkoutsos, L. Bello, Y. Ben-Haim, D. Bucher, F. J. Cabrera-Hernádez, J. CarballoFranquis, A. Chen, C.-F. Chen, J. M. Chow, A. D. CórcolesGonzales, A. J. Cross, A. Cross, J. Cruz-Benito, C. Culver, S. D. L. P. González, E. D. L. Torre, D. Ding, E. Dumitrescu et al., QISKIT: An open-source framework for quantum computing (2019).

[35] A. W. Chin, J. Prior, S. F. Huelga, and M. B. Plenio, Generalized Polaron Ansatz for the Ground State of the Sub-Ohmic Spin-Boson Model: An Analytic Theory of the Localization Transition, Phys. Rev. Lett. 107, 160601 (2011).

[36] J. C. Spall et al., Multivariate stochastic approximation using a simultaneous perturbation gradient approximation, IEEE Trans. Autom. Control 37, 332 (1992).

[37] S. Chilingaryan and B. Rodríguez-Lara, Exceptional solutions in two-mode quantum rabi models, J. Phys. B: At., Mol. Opt. Phys. 48, 245501 (2015).

[38] B. Vlastakis, A. Petrenko, N. Ofek, L. Sun, Z. Leghtas, K. Sliwa, Y. Liu, M. Hatridge, J. Blumoff, L. Frunzio et al., Characterizing entanglement of an artificial atom and a cavity cat state with bell's inequality, Nat. Commun. 6, 8970 (2015).

[39] A. Smith, M. S. Kim, F. Pollmann, and J. Knolle, Simulating quantum many-body dynamics on a current digital quantum computer, npj Quantum Inf. 5, 106 (2019).

[40] R. Sagastizabal, X. Bonet-Monroig, M. Singh, M. A. Rol, C. C. Bultink, X. Fu, C. Price, V. P. Ostroukh, N. Muthusubramanian, A. Bruno, M. Beekman, N. Haider, T. O'Brien, and L. DiCarlo, Error mitigation by symmetry verification on a variational quantum eigensolver, Phys. Rev. A 100, 010302 (2019).

[41] R. Silbey and R. A. Harris, Variational calculation of the dynamics of a two level system interacting with a bath, J. Chem. Phys. 80, 2615 (1984).

[42] J. D. Whitfield, J. Biamonte, and A. Aspuru-Guzik, Simulation of electronic structure hamiltonians using quantum computers, Mol. Phys. 109, 735 (2011).

[43] K. Temme, S. Bravyi, and J. M. Gambetta, Error Mitigation for Short-Depth Quantum Circuits, Phys. Rev. Lett. 119, 180509 (2017).

[44] J. R. Johansson, P. D. Nation, and F. Nori, Qutip 2: A python framework for the dynamics of open quantum systems, Comput. Phys. Commun. 184, 1234 (2013).

[45] D. C. McKay, S. Filipp, A. Mezzacapo, E. Magesan, J. M. Chow, and J. M. Gambetta, Universal Gate for Fixed-Frequency Qubits via a Tunable Bus, Phys. Rev. Appl. 6, 064007 (2016). 
[46] A. Blais, J. Gambetta, A. Wallraff, D. I. Schuster, S. M. Girvin, M. H. Devoret, and R. J. Schoelkopf, Quantum-information processing with circuit quantum electrodynamics, Phys. Rev. A 75, 032329 (2007).
[47] P. J. Liebermann, P.-L. Dallaire-Demers, and F. K. Wilhelm, Implementation of the ifredkin gate in scalable superconducting architecture for the quantum simulation of fermionic systems, arXiv:1701.07870. 